\title{
MAPK14-TFEB pathways promote microglia activation through inhibiting CMA-mediated NLRP3 degradation in Parkinson's disease
}

\section{Jialong Chen}

Southern Medical University

Kanmin Mao

Southern Medical University

Honglin Yu

Southern Medical University

Yue Wen

Southern Medical University

\section{Hua She}

Emory University School of Medicine

\section{Liuna Yang}

Guangdong Medical College Zhanjiang Campus: Guangdong Medical University

He Zhang

Guangdong Medical College: Guangdong Medical University

\section{Huihui Li}

Southern Medical University

\section{Xian Wu}

Southern Medical University

\section{Yixian Ren}

Southern Medical University

wenjun li ( $\nabla$ cjlwj@smu.edu.cn )

Southern Medical University https://orcid.org/0000-0003-1152-5016

Fei Zou

Southern Medical University

\section{Research article}

Keywords: Parkinson's disease, MAPK14, NLRP3, Chaperone-mediated autophagy, TFEB

Posted Date: December 22nd, 2020

DOI: https://doi.org/10.21203/rs.3.rs-131694/v1 
License: (c) (i) This work is licensed under a Creative Commons Attribution 4.0 International License. Read Full License 


\section{Abstract}

Background Parkinson's disease (PD) is characterized by the degeneration of dopaminergic neurons in the substantia nigra pars compacta (SNpc), accompanied by chronic neuroinflammation, autophagy dysfunction and SNCA(a-synuclein) accumulation in the form of Lewy bodies. Previous studies show that misfolded SNCA upregulate the inflammatory cascade through NOX activation, which elevated ROS production in microglial cell. However, it's still not elucidated about the molecular mechanisms between autophagy deficiency and neuroinflammation induced by SNCA accumulation in microglia.

Methods We overexpress mice and BV2 cells with SNCAA53T mutant as models of early-onset Parkinson's disease and identified the mechanisms that MAPK14-TFEB pathways promote microglia activation through inhibiting CMA-mediated NLRP3 degradation in Parkinson's disease by a series of methods of molecular biology. The results of pathology and animal behavior showed that the inhibition of MAPK14 and NLRP3 had protective effect on Parkinson's disease model.

Results Here, we report that MAPK14 (p38a) activates NLRP3 inflammasome via inhibiting TFEB in BV2 cells. Transcription Factor EB (TFEB) signaling negatively regulates NLRP3 inflammasome through increasing LAMP2A expression, which binds to NLRP3 and promotes its degradation via chaperonemediated autophagy(CMA). Importantly, both MAPK14 inhibitor SB203580 and NLRP3 inhibitor MCC950 not only prevents neurodegeneration in vitro, but also alleviates movement impairment and SNCA/asynuclein abnormal accumulation in SNCA ${ }^{\mathrm{A} 53 \mathrm{~T}}$-tg mice model of Parkinson's disease.

Conclusion Our research reveals an endogenous regulatory mechanism of NLRP3 turnover and microgliadopaminergic neuron interaction, which may be a potential therapeutic strategy for Parkinson's disease.

\section{Background}

Parkinson's disease (PD) is characterized by the loss of dopaminergic neurons in the substantia nigra, resulting in neuromuscular dysfunction such as rest tremor. The mitochondrial dysfunction, calcium dyshomeostasis, chronic inflammation and oxidative stress are contributed to the molecular pathogenesis of PD. Previous studies shown that neuroinflammation may be the primary factor leading to the process of PD-related pathogenesis and causing the degeneration and loss of dopaminergic neurons. Proinflammatory cytokine such as tumor necrosis factor $\alpha$ and IL-1 $\beta$ were found to be upregulated in the cerebrospinal fluid and blood of PD patients ${ }^{1}$. Moreover, activated microglia releases proinflammatory cytokines which lead to neuronal death. Abnormal accumulation of SNCA causes neuroinflammation, in turn, neuroinflammation exacerbates dopaminergic neurons loss. This vicious circle closely associated with the pathological process of PD. The NLRP3 inflammasome was originally defined by Tschopp that activates inflammatory caspases and release mature IL-1 $\beta$ and IL-18 ${ }^{2}$. The NLRP3 inflammation complex contributes to the activation of the innate immune response and causes cell pyrotosis ${ }^{2,3}$. Recent studies demonstrated that NLRP3 inflammasome is involved in the progression of neurodegenerative disease and NLRP3 deficiency plays a protective role in animal models of 
Alzheimer's disease (AD) and PD ${ }^{4,5}$. Although the mechanisms that NLRP3 inflammasome participates in diverse inflammatory diseases has been extensively investigated, its regulatory networks in microglia are unclear.

Mitogen-activated protein kinases (MAPKs) regulate various cellular processes. The p38 mitogenactivated protein kinase, a prominent member of the MAPKs, not only involved in cell cycle, cell death, development, differentiation, senescence and tumorigenesis, but also functions as a specific class of serine/threonine kinase regulating the inflammation responses. The important role of MAPK14-mediated inflammatory responses was originally confirmed as the target of the pyridinyl imidazole that inhibited inflammatory cytokines release in LPS-treated monocytes ${ }^{6}$. The MAPK pathways contributed to the increased production of proinflammatory cytokines by microglia that treated with toll-like receptor (TLR) ligands or beta-amyloid $(A \beta)^{7}$. Recently, Mao and colleagues identified that MAPK14 inhibits autophagy and promotes microglial inflammatory responses via ULK1 phosphorylation ${ }^{8}$. A few studies have demonstrated that pharmacological inhibition of MAPK14 plays a protective role in several animal models of neurodegenerative disease ${ }^{9-11}$. Moreover, studies in vivo have also shown that MAPK14 deficiency plays a protective role in $A D$ via enhancing the level of autophagy ${ }^{12,13}$. Although the protective effect of MAPK14 inhibition and its implication in neurodegenerative diseases are emerging, the mechanisms by which MAPK14 regulates inflammatory responses in microglia remains elusive.

Here, we demonstrate that MAPK14-TFEB pathway regulates NLRP3 inflammasome degradation via CMA. We identify that NLRP3 is a novel substrate for CMA and MAPK14 inhibits TFEB activity via phosphorylation which blocks NLRP3 degradation. Besides, our results reveal that both MAPK14 inhibitor SB203580 and NLRP3 inhibitor MCC950 reduced SNCA-induced microglia activation and dopaminergic neuronal loss. All the results suggest the MAPK14-TFEB pathway may be a potential treatment target for NLRP3 inflammasome-driven neurodegenerative diseases.

\section{Materials And Methods}

Animal. The SNCA ${ }^{\text {A53T }}$-tg mice expressing mutant A53T SNCA (the 140 amino acid isoform) under the direction of the mouse prion protein promoter has been described previously ${ }^{26}$. The SNCA ${ }^{\text {A53T }}$-tg mice expressed mutant human A53T SNCA were purchased from Model Animal Research Center of Nanjing University. The structure, location and onset of the inclusions seen in the mutant mice resemble characteristics seen in human neuronal a-synucleinopathies and the transgenic mice are generally used in the study of Parkinson's Disease. Animals were individually housed under light: dark (12:12 h) cycles and provided with food and water.

Balance beam test. Mice were placed on a narrow beam $(20 \mathrm{~mm} / 12 \mathrm{~mm})$ suspended $20 \mathrm{~cm}$ above soft bedding, and their movement from one end towards the other end was recorded. The number of missteps (paw faults, or slips) was scored during the trip. 
Pole test. This test was performed based on the method described by Ogawa ${ }^{27}$ used as a measure for bradykinesia. Mice ( $n=8$ in each group) were placed the top of a pole $(55 \mathrm{~cm}$ in height, $10 \mathrm{~mm}$ in diameter) with a rough surface. Both the time to turn and climb down was recorded.

Administration of the MAPK14 inhibitor SB203580 and MCC950. All animal procedures were approved by the ethics committee of the Southern Medical University. All groups of animals received the inhibitor SB203580 (5 mg/ $\mathrm{kg})$ and MCC950 (10 mg/ $\mathrm{kg})$ through intraperitoneal injection. In each experimental cohort, male mice were randomly matched for group assignment. SNCA ${ }^{\mathrm{A} 53 \mathrm{~T}}$-tg mice were injected with the drug starting from 5 months of age until the end of 9 months. Animals were sacrificed after the final injection. The midbrain and cortex of half mice were dissected and used for Western blotting analysis. Meanwhile, the other half mice were perfused with $4 \%$ paraformaldehyde and paraffinized coronal sections were processed for immunohistochemistry assay.

Plasmids, Antibody and chemicals. EGFP-SNCA A53T (40823), PHM6-SNCA-A53T (40825), pAAVSNCA WT (36055), pCDNA3 Flag p38a (20352), and pMT3-p38 (12658) were obtained from Addgene. The following antibodies were used: anti-Parkin (Cell Signaling Technologies, \#4211), anti-LC3A/B (Cell Signaling Technologies, \#12741), anti-beta Tubulin (Cell Signaling Technologies, \#2146), anti-MAPK14 (Abcam, ab170099), anti-p-p38tyr182 (Santa Cruz Biotechnology, Santa Cruz, CA, USA), antiSNCA(Proteintech,10842-1-AP), anti-synapsin-1 (Cell Signaling Technologies, \#5297), antiNLRP3(Novus,NBP2-12446), anti-IL-1 $\beta$ (Abcam,ab9722), anti-TFEB(Proteintech,13372-1-AP), antiTH(Santa,sc-25269), anti-ACTB(CellSignaling Technologies; \#4970), anti-LaminB (Proteintech,12987-1AP), anti-SQSTM1/P62(Proteintech, 18420-1-AP), anti-GAPDH(Proteintech, 10494-1-AP), antiCASP1(Proteintech, 22915-1-AP), anti-HSPA8(Proteintech, 66442-1-lg), anti-ASC(Cell Signaling Technologies, \#67824), anti-LAMP2A(Proteintech, 66301-1-Ig), anti-IBA-1(Cell Signaling Technologies, \#17198), anti-GFP(Proteintech, 50430-2-AP), anti-14-3-3(Cell Signaling Technologies, \#95422), antiFLAG(Proteintech, 66008-3-lg). ELISA KITs were purchased from MEIMIAN (Guangzhou, China) including CASP1(MM-0820M2) and IL-1ß(MM-0040M2). SB203580 (Selleck, S1076) and MCC950 (Selleck, S7809) were purchased from Selleckchem (Houston, TX, USA). The siRNA for MAPK14 was: 5 ' -

GGUCUGUUGGAUGUGUUCAdTdT-3'. PCR primers of a-synucleinA53T-tg were: F 5'-

TGTAGGCTCCAAAACCAAGG-3', R 5'-TGTCAGGATCCACAGGCATA-3.

Immunohistochemistry. The paraffin sections of brain tissue were collected for routine immunohistochemistry staining for p-MAPK14 (1:50), TH (1:100), IBA-1 (1:100), IL-1 $\beta$ (1:100), NLRP3(1:100), LAMP2A (1:100) and TFEB (1:100). The area of the midbrain dopaminergic neurons from brain sections according to the anatomical position and the location of $\mathrm{TH}$-positive neurons has been described previously ${ }^{28}$. Immunohistochemistry staining was assessed by two independent pathologists with no prior knowledge of patient characteristics. In order to describe the image where were taken, we provided the model of anatomical position of brain section and the lower power image of TH-positive immunohistochemical brain section. As figure S3 A and S3 B shown that the image of SNpc were taken according to TH positive location and the image of cortex were taken according to their anatomical location. The percentage of positive cells and the expression levels of proteins in the brain tissue were 
scored according to intensity as previously described. Specifically, percentages $\leq 1 \%, 2 \%-25 \%, 26 \%-50 \%$, $51 \%-75 \%$ and $\geq 75 \%$ were scored as $0,1,2,3$ and 4, respectively; non-significant brown, light brown, moderate brown and deep brown staining intensities were scored as $0,1,2$ and 3 , respectively. Combined scoring was then calculated and graded as negative $(-; 0-1)$, weak $(+; 2-4)$, moderate $(++; 5-8)$, and strong $(+++; 9-12)$.

Transmission electron microscopy (TEM). SN4741 cells were washed once with PBS, collected with a cell scraper. The sections of midbrain tissue were collected and washed once with cold PBS. Cell and midbrain tissue pellets were resuspended in the fixative containing $2.5 \%$ glutaraldehyde in PBS at $4{ }^{\circ} \mathrm{C}$. Cell and midbrain tissue pellets were fixed for $3 \mathrm{~h}$. After rinsing with sodium cacodylate buffer, they were further fixed in $1 \%$ OsO4 in sodium cacodylate buffer on ice for $1 \mathrm{~h}$ and dehydrated with acetone, and then embedded in resin and polymerized at $60^{\circ} \mathrm{C}$ for $48 \mathrm{~h}$. Ultrathin sections were obtained on an ultramicrotome and stained with uranyl acetate and lead citrate before observation under Hitachi $\mathrm{H}-7500$ TEM.

Cell culture and transfection. SN4741 cells were cultured in DMEM medium containing 1\% glucose, 100 $\mathrm{U} / \mathrm{ml}$ penicillin-streptomycin, $1 \% \mathrm{~L}$-glutamine and supplemented with $10 \%$ fetal bovine serum at $33^{\circ} \mathrm{C}$. BV2 cells were cultured in DMEM medium supplemented with $10 \%$ fetal bovine serum at $37^{\circ} \mathrm{C}$.Cells were transfected with Lipofectamine 3000 reagent (Invitrogen, Carlsbad, CA, USA) following the manufacturer's instructions.

Western blotting and co-immunoprecipitation. Cytosolic and nuclear fractions were collected using an isolation kit (KeyGEN, Nanjing, China) following the manufacturer's instructions. For Western blotting, cells were collected in RIPA buffer containing $20 \mathrm{mM}$ Tris- $\mathrm{HCl}, \mathrm{pH} 7.4,150 \mathrm{mM} \mathrm{NaCl}$ and 1\% Triton X-100 with protease inhibitor cocktail (Roche, Nutley, NJ, USA). The extracts were centrifuged for $10 \mathrm{~min}$ at $13,000 \mathrm{rpm}$ at $4{ }^{\circ} \mathrm{C}$, and the supernatants were used for immunoblot analysis. For coimmunoprecipitation, cells were centrifuged at $13,000 \mathrm{~g}$ for $10 \mathrm{~min}$ at $4{ }^{\circ} \mathrm{C}$, and then the supernatant was transferred into a new tube. And then $5 \mu \mathrm{g}$ of the corresponding first antibody was added into the samples and incubating overnight at $4{ }^{\circ} \mathrm{C}$. On the next day, $50 \mu$ agarose beads were added into the samples and incubating $2 \mathrm{~h}$ at $4^{\circ} \mathrm{C}$. The immunocomplex was collected with centrifugation at $1,500 \mathrm{~g}$ for $1 \mathrm{~min}$ at $4{ }^{\circ} \mathrm{C}$. And washed 3 times with RIPA buffer. Proteins were eluted from beads with $2 \times$ SDS loading buffer and subjected to immunoblot analysis. The immunoreactive bands were detected by Odyssey Infrared Imaging System. The band intensity was analyzed with Image J. Antibodies used in Western blotting are diluted by 1000 , except TH (1:100) and p-MAPK14(1:100).

Apoptosis and cell death. Cell apoptosis of SN4741 cell was measured using a Cell Apoptosis Kit (Dojindo, Japan) according to the manufacturer's instructions. Percent of cell apoptosis was determined by staining with $2 \mu \mathrm{M}$ Annexin $\mathrm{V}$ and $2 \mu \mathrm{M}$ Propidium lodide (PI). The number of cell apoptosis was counted by flow cytometry and images of apoptosis was taken by Laser Scanning Confocal Microscopy.

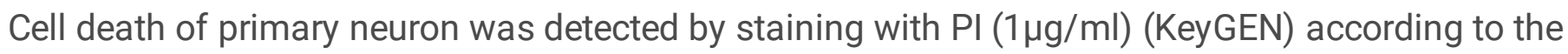


manufacturer's instructions. Images were captured by fluorescence microscope and analyzed by Image $\mathrm{J}$ analysis software.

Measurement of mitochondrial membrane potential. The mitochondrial membrane potential in cells was assessed using Tetramethylrhodamine methyl ester (TMRE; Thermo Fisher, T669). Cells were washed with $1 \times$ PBS and then incubated with TMRE $(0.1 \mu \mathrm{M})$ in medium for $20 \mathrm{~min}$ in the dark. The intensity of fluorescence was monitored using fluorescence microscope.

Linear range of detection in quantitative Western blot analysis. Determining the Linear Range for a Target Protein and Housekeeping Protein (HKP as loading control). First, performing a Western blot with a serial dilution of sample (an 8-12 point) and detecting target protein and HKP by Near-Infrared Western Blot Detection Protocol (LI-COR). Then, Empiria Studio ${ }^{\text {TM }}$ Software be used to detect the linear range. Empiria Studio $^{T M}$ Software can generate the combined linear graph. For experiments, choose a level of sample loading that falls in the middle of the combined linear range for the target protein and HKP. Avoid working near the boundaries of the combined range. Whenever an experimental parameter is changed (such as sample type, treatment conditions, transfer method, membrane, antibody, or detection reagents), the combined linear range must be re-validated.

ELISA. Tissue specimen: After cutting the specimen, weigh $0.1 \mathrm{~g}$ of tissue, add $9 \mathrm{ml}$ of PBS with $\mathrm{pH} 7.2-$ 7.4, and homogenize the specimen. Centrifuge for about 20 minutes (2000-3000 rpm) and carefully collect the supernatant that used for ELISA detection following the manufacturer's instructions. Cell culture medium: Cell culture medium was centrifuged and used for ELISA detection following the manufacturer's instructions.

Immunofluorescence. Cells were grown on a confocal dish for 2 days, and then cells were washed with PBS three times, then fixed with $4 \%$ paraformaldehyde for $15 \mathrm{~min}$ at room temperature. After that, cells were permeabilized with frozen methanol for $10 \mathrm{~min}$ at $-20^{\circ} \mathrm{C}$ and blocked in $5 \%$ BSA for $30 \mathrm{~min}$. And then samples were incubated with primary antibody (1:100 1:200) in 5\% BSA for overnight at $4^{\circ} \mathrm{C}$, and then incubated with secondary antibody $(1: 100)$ in $5 \%$ BSA for $1 \mathrm{~h}$ at room temperature. An Olympus FV1000 confocal microscope with a 100x objective was used for image capture. The colocalization signal was analyzed with Image Pro Plus software.

Statistical analysis. All data of experiments were analyzed with GraphPad Prism 7 software (La Joya, Ca, USA). Data from Western blot and immunohistochemistry analysis were performed with the test for two groups or ANOVA with Tukey's post-test (GraphPad Software) for multiple groups. Data from Balance beam test, Pole test, Transmission electron microscopy, Immunofluorescence, were analyzed by two-way ANOVA followed by Tukey's post-test. All values are expressed as the mean \pm SEM, and $p$ values $<0.05$ were considered statistically significant. All experiments were repeated at least 3 times.

\section{Results}

\section{NLRP3 inflammasome is activated in $\mathrm{SNCA}^{\mathrm{A} 53 \mathrm{~T}}$-tg mice}


In order to investigate the effects of SNCA accumulation and related toxicity, we used SNCA A53T transgenic mice (SNCA ${ }^{\mathrm{A53T}}$-tg mice). The accumulation of SNCA was identified in both the SNpc and the cortex at 9 months of age (Fig.S1 A). To determine the effect of SNCA on NLRP3 inflammasome activation, we measured the expression of core components of this multiprotein complex of the canonical inflammasome in both the SNpc and the cortex of SNCA ${ }^{A 53 T}$-tg mice by $\mathrm{IHC}$ and Western blotting. In the brain sections of the SNpc and the cortex, NLRP3 inflammasome activation was remarkably intensified, as evidenced by increased NLRP3 expression as well as over-production of IL-1 3 (Fig.1 A-D). Results of Western blotting revealed that the protein level of NLRP3, ASC, cleaved CASP1 (caspase1) and cleaved IL$1 \beta$ were increased both in SNpc and cortex of SNCA ${ }^{A 53 T}$-tg mice, which further demonstrated that the NLRP3 inflammasome was activated in SNCA ${ }^{\mathrm{A} 53 \mathrm{~T}}$-tg mice (Fig.1 E-G). Besides, microglial activation, as assessed morphologically by immunohistochemistry with the classic antibody specific for Iba-1, microglia was activated in SNCA ${ }^{A 53 T}$-tg mice brain both of the SNpc and the cortex (Fig.S1 B). To further confirm this result, PD patients' serum was assayed by ELISA. Consistently, over-production of IL-1 $\beta$ and IL-18 were found in PD patients' serum (Fig.S1 C-D). Collectively, these data suggest that the NLRP3 inflammasome is activated in the pathological process of PD.

\section{NLRP3 inflammasome activation is suppressed by MAPK14 inhibitor SB203580}

To examine whether MAPK14 involved in the NLRP3 inflammasome activation in PD, we first investigated the levels of MAPK14 phosphorylation in the SNpc and the cortex by IHC. Results showed that compared with wild-type mice, MAPK14 was activated in both the SNpc and the cortex of SNCA ${ }^{\mathrm{A} 53 \mathrm{~T}}$-tg mice at 9 months of age (Fig.2 A, B). Western blotting also identified increased phosphorylated MAPK14 and SNCA accumulation in the SNpc and the cortex of SNCA ${ }^{\mathrm{A} 53 \mathrm{~T}}$-tg mice (Fig.2 C, D, E).

To assess whether NLRP3 activation is mediated by MAPK14, SNCA ${ }^{\mathrm{A} 53 \mathrm{~T}}$-tg mice were injected with MAPK14 inhibitor SB203580 starting at the age of 5 months until the end of 9 months (Fig.S2 A). Then, we analyzed NLRP3 and IL-1 $\beta$ by IHC. The results showed that SB203580 significantly reduced the levels of NLRP3 and IL-1 $\beta$ both in SNpc and cortex of SNCA ${ }^{A 53 T}$-tg mice (Fig.3A-D). Furthermore, the increased protein levels of NLRP3, ASC, cleaved CASP1, and cleaved IL-1 $\beta$ in SNCA ${ }^{\mathrm{A} 53 \mathrm{~T}}$-tg mice (Fig.3 E-G) and BV2A53T cells were abolished after SB203580 treatment as detected by Western blotting(Fig.S2 B, C). Also, we have detected the Caspase 1 activity as well as mature IL-1 $\beta$ using ELISA in the tissue homogenates and results revealed that SB203580 can decrease the levels of caspase 1 activity and mature IL1 $\beta$ in SNCA $^{\text {A53T }}$-tg mice (Fig.S2 D, E). Of note, SB203580 did not affect the transcription of SNCA mRNA (Fig.S2 F). Together, these data suggested that MAPK14 inhibitor SB203580 reduced neuroinflammation caused by SNCA accumulation.

\section{SB203580 induces NLRP3 degradation via chaperone-mediated autophagy}

The activation of NLRP3 inflammasome in cells is tightly regulated. Excessive activation of the NLRP3 inflammasome is involved in the pathological process of various diseases including PD. To test whether NLRP3 is degraded by CMA, the interaction between NLRP3 and LAMP2A was examined by 
immunoprecipitation in brain tissues. The results showed that LAMP2A interacts with NLRP3 in vivo (Fig.4 A). Moreover, we also found that SB203580 enhanced the NLRP3/LAMP2A and NLRP3/ HSPA8 interaction in BV2 cells (Fig.4 B, C).

In CMA, the substrate usually contains a KFERQ-like pentapeptide consensus sequence that is recognized by HSPA8 (HSC70). Inspection of the amino acid sequence of mouse NLRP3, two KFERQ-like motifs, 795QKLVE799 and 989EVLKQ993, were revealed (Fig.S3 A). To determine whether NLRP3 is a bona fide substrate for CMA, the first two amino acids of the mouse NLRP3 KFERQ-like sequence were mutated to alanine (NLRP3AA). The result showed that mutation of the KFERQ-like sequence of NLRP3 abolished its interaction with HSPA8 (Fig.4 D, E).

To further assess whether CMA is involved in the regulation of NLRP3, we treated BV2 cells with serum deprivation or AR7 $(10 \mu \mathrm{M}, 24 \mathrm{~h})$ or QX77 $(10 \mu \mathrm{M}, 24 \mathrm{~h})$, the latter two chemicals are well-known CMA agonists. The results showed that both serum deprivation and CMA agonists (AR7, QX77) increased the levels of LAMP2A and reduced the levels of NLRP3 (Fig.4 F-I). Moreover, the level of LAMP2A in the SNpc of mice was analyzed. Treatment with MAPK14 inhibitor SB203580 elevated the level of LAMP2A, a key receptor for CMA (Fig.4 J, K).

\section{SB203580 activates TFEB-mediated autophagy}

Our data in Figure 2 and 3 demonstrate that MAPK14 mediates the NLRP3 inflammasome activation in BV2 cells and in SNCA ${ }^{\text {A53T }}$-tg mice, as evidenced by increased levels of NLRP3, cleaved CASP1, and IL-1 3 . Furthermore, in Figure 4, we showed that MAPK14 regulates NLRP3 turn over through CMA. All these prompt us to examine the role of MAPK14 in all over autophagy regulation, which may subsequently contribute to the NLRP3 inflammasome activation ${ }^{14}$. TFEB is a master protein for lysosomal biogenesis. In SNpc sections from SNCA ${ }^{A 53 T}$-tg mice, the total levels of TFEB and LAMP1 were decreased, while the SQSTM1/p62 level was increased (Fig.5 A, B). To determine whether SNCA aggregation affects the TFEB nuclear translocation, subcellular localization of TFEB was examined by sub-cellular fractionation. The result showed that the nuclear TFEB level decreased in SNpc sections of SNCA ${ }^{\mathrm{A} 53 \mathrm{~T}}$-tg mice (Fig.S4 A, B).

In order to examine whether MAPK14 activation is involved in TFEB-mediated autophagy in the PD model, we treated SNCA-A53T overexpressing BV2 cells with MAPK14 inhibitor SB203580 (10 $\mu \mathrm{M}, 24 \mathrm{~h})$. The results showed that MAPK14 inhibitor SB203580 significantly elevates TFEB-mediated autophagy, as evidenced by the increased LAMP1, MAP1LC3/LC3 and the decrease of SQSTM1 (Fig.5 C, D). Furthermore, both Western blotting and immunofluorescence revealed that SB203580 prompted TFEB nuclear translocation from the cytoplasm (Fig.5 E-H). Meanwhile, SB203580 enhanced the biogenesis of lysosomes (Fig. S4 C, D). Besides, SB203580 also increased the level of TFEB in SNpc of mice as detected by IHC (Fig.5 I, J). All these data suggested that SB203580 promotes TFEB-mediated autophagy.

\section{MAPK14 interacts with and phosphorylates TFEB at serine 211}


Next, we aimed to elucidate the mechanism by which MAPK14 regulates TFEB-mediated autophagy. To evaluate whether TFEB could be a substrate of MAPK14, we first detected the interaction between MAPK14 and TFEB by co-immunoprecipitation. As expected, immunoprecipitation analysis showed that MAPK14 interacted with TFEB both in BV2 cells and mice brain (Fig.6 A-E). Of note, SB203580 reduced the MAPK14/TFEB interaction that enhanced by SNCA A53T aggregation (Fig.6 B-E).

To identify the regulatory function of MAPK14 on TFEB, we examined the role of MAPK14 on the interaction between TFEB and14-3-3 proteins. As phosphorylation of TFEB at serine 211 by mTORC1 has been proved to promote TFEB binding to 14-3-3, we detected the level of TFEB phosphorylation with a phospho-Ser211 specific antibody. It showed that SB203580 reduced the TFEB phosphorylation at serine 211(Fig.6 F, G). To explore the mechanism by which MAPK14 regulates TFEB's function, we analyzed the effect of overexpression kinase-dead MAPK14 mutant on TFEB's nuclear translocation. Compared with MAPK14 WT, kinase-dead MAPK14 overexpression increased the nuclear translocation of TFEB (Fig. $6 \mathrm{H}$, I) and enhanced the biogenesis of lysosome (Fig. $6 \mathrm{~J}, \mathrm{~K}$ ). Meanwhile, kinase-dead MAPK14 overexpression declined the levels of NLRP3, ASC, C-CASP1 detected by WB (Fig.6 L, M). To further confirm the effects from p38alpha-MAPK (MAPK14), we analyzed the effect of MAPK14 siRNA. As figure S 5 shown, MAPK14 siRNA increased the biogenesis of lysosome (Fig. S 5A-B). Also, MAPK14 siRNA decreased the levels of NLRP3, C-CASP1 detected by WB. (Fig. S 5 C, D). These results supported that MAPK14 activation suppresses TFEB function via phosphorylating TFEB at serine 211 and inhibiting TFEB nuclear translocation.

These finding including the interaction between MAPK14 and TFEB, the inhibitory effects of MAPK14 inhibitor and the activation of NLRP3 inflammasome regulated by autophagy, raise the possibility that the MAPK14 may curb TFEB to increase NLRP3 inflammasome activation. To test the hypothesis, we firstly overexpressed TFEB in SNCA-A53T BV2 cells. The results showed that TFEB overexpression reduced NLRP3 and cleaved CASP1 as detected by Western blotting (Fig. S 6 A, B). To examine whether MAPK14 activates NLRP3 via regulating TFEB, we compared the effects of SB203580 treatment, TFEB knockdown, and both SB203580 treatment and TFEB knockdown in SNCA-A53T BV2 cells. Remarkably, the overactivation of NLRP3 inflammasome and cleaved CASP1 resulted from SNCA-A53T was almost completely reversed by SB203580, while TFEB knockdown eliminated the effect of SB203580 (Fig. S 6 C, D). Similarly, the effect of SB203580 in IL-1 $\beta$, IL-18 were abrogated in SNCA-A53T BV2 cells with TFEB knockdown (Fig. S 6 E-F). To further test whether the autophagy/lysosome pathway is responsible for MAPK14 inhibition induced NLRP3 degradation, we treated SNCA-A53T BV2 cells with autophagy/lysosome inhibitor CQ $(10 \mu \mathrm{M}, 4 \mathrm{~h})$. The results showed that autophagy/lysosome inhibitor CQ blocked SB203580-induced NLRP3 degradation (Fig. S 6 G, H). These results supported that MAPK14 activates NLRP3 inflammasome via disturbing TFEB-mediated autophagy.

\section{SNCA-induced NLRP3 activation in microglia promoted dopaminergic neuronal loss}

To examine whether microglial activation is responsible for the death of dopaminergic neurons, SN4741 cells were treated with conditioned medium form microglia. After appropriate treatments, Cell death in 
SN4741 cells were determined by flow cytometry. As figure 7 A-B shown, conditioned medium form A53T SNCA microglia caused dopaminergic neuronal loss, while NLRP3 inhibitor MCC950 (10 $\mu \mathrm{M}, 12 \mathrm{~h})$ and MAPK14 inhibitor SB203580 can effectively eliminate the effect.

To address whether NLRP3 activation is involved in dopaminergic neuronal loss in vivo, SNCA ${ }^{\mathrm{A} 53 \mathrm{~T}}$-tg mice were fed with NLRP3 inhibitor MCC950 starting at the age of 5 months until the end of 9 months (Fig.S7 A). Interestingly, MCC950 significantly inhibited the activation of microglia in SNCA ${ }^{\text {A53T }}$-tg mice, which suggests that NLRP3 plays a key role in the activation of microgliain PD(Fig7 C-D). Then, we analyzed the levels of TH in SNpc by WB and IHC. The results showed that MCC950 significantly reduced the levels of dopaminergic neuronal loss in SNpc of SNCA ${ }^{A 53 T}$-tg mice (Fig. $7 \mathrm{E}-\mathrm{H}$ ). Furthermore, Balance beam test and pole test showed that MCC950 treatment significantly improved the motor activity in SNCA $^{\text {A53T }}$-tg mice (Fig. S 7 B, C).

\section{SB203580 plays a protective role in $\mathrm{SNCA}^{\mathrm{A} 53 \mathrm{~T}}$-tg mice}

The above data showed that MAPK14-TFEB pathway is involved in NLRP3 inflammasome activation, which prompts us to test whether MAPK14 inhibitor SB203580 has a protective role in SNCA ${ }^{\text {A53T }}$-tg mice. Wild type and SNCA ${ }^{\mathrm{A} 53 \mathrm{~T}}$-tg mice were treated with or without the MAPK14 inhibitor SB203580. Balance beam test and Pole test showed that SB203580 treatment significantly improved the motor activity in

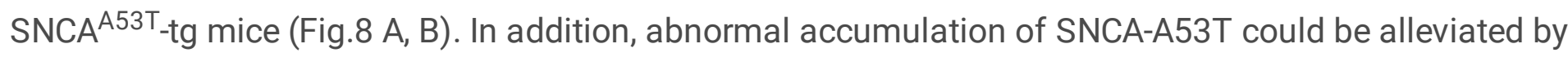
MAPK14 inhibition (Fig.8 C-F). Synapse loss correlates with cognitive impairment in neurodegenerative diseases including $A D$ and $P D^{15,16}$. To examine the protective effect of MAPK14 inhibition, we detected the presynaptic proteins synapsin-1 (SYN1) in SNCA ${ }^{\text {A53T }}$-tg mice. The results showed that SB203580 rescues the reduced level of SYN1 in SNCA ${ }^{\text {A53T }}$-tg mice (Fig.8 G, H). Under TEM, SNCA ${ }^{A 53 T}$-tg mice have fewer synaptic vesicles, while SB203580 treatment partially restores synaptic vesicle loss (Fig.8 I, J). Taken together, these data indicated that MAPK14 inhibition provides protection in SNCA ${ }^{\text {A53T }}$-tg mice.

\section{Discussion}

Parkinson Disease (PD) is a common, age-related neurodegenerative disease afflicting more than 2-3\% of people over the 65 and more than $4 \%$ of the population by the age of 85 . The progressive loss of dopaminergic neurons in the substantia nigra (SN) eventually results in motor alterations of PD. The precise mechanism underlying the pathogenesis of PD is not yet been fully elucidated. Previous studies shown the defect of autophagy-lysosomal pathway (ALP) and neuroinflammation are contributor in the PD pathophysiology. Accumulating evidence suggests that immune signaling cascades are regulated by autophagy, while the mechanisms that modulate the inflammasome activation process is still unclear. In our study, NLRP3 was identified as a novel chaperone-mediated autophagy (CMA) substrate and activation of CMA promotes NLRP3 degradation and thereby inhibits overproduction of proinflammatory cytokines in microglia. Moreover, we demonstrated that MAPK14 activation caused by SNCA A53T inhibits TFEB-mediated CMA, which promotes NLRP3 inflammasome activation. Furthermore, we found 
that both MAPK14 and NLRP3 inhibitor can reduce SNCA-induced microglia activation and dopaminergic neuronal loss. Therefore, MAPK14-TFEB pathways regulates NLRP3 degradation via CMA in SNCAinduced microglia activation, which promotes dopaminergic neuronal loss.

The chronic neuroinflammation in PD has been intensively investigated in the past decade, but the precise origin of the CNS inflammation response remains unclear. Evidences found that neuroinflammatory response is a key factor in the pathological process of $\mathrm{PD}^{17}$. Activated microglia and increased levels of pro-inflammatory factors were identified in postmortem analysis and PD models. SNCA abnormal aggregation can appears in neuron and microglia, which not only disrupts neuronal function, also causes activation of microglia that increase phagocytic activity and pro-inflammatory cytokines production ${ }^{18}$. It is still controversial whether SNCA can trigger microglia responses and pathological reaction. Our results not only identified that NLRP3 was a novel CMA substrate, but also revealed that MAPK14 activation caused by SNCA inhibits TFEB activity that blocked CMA, and then decreased NLRP3 degradation in microglia. Studies found that SNCA abnormal aggregation activated microglia, which led to death of dopaminergic neurons in the substantia nigra of the midbrain ${ }^{19}$. Toll-like receptor 2 (TLR2) of microglia was identified as the receptor for secreted SNCA, which transport SNCA into microglial cytoplasm ${ }^{20}$. Overexpression of a-synuclein increases microglial activation in vivo. However, SNCA internalization still appears when TLR2 is deficient, suggesting a-synuclein uptake relies on multiple receptor systems ${ }^{21}$.

The activation of NLRP3 inflammasome usually requires upregulation of the transcription of NLRP3 and pro-IL-1 $\beta$ by NF-KB. However, recent studies report that NLRP3 inflammasome can be regulated independently of transcription. For instance, lys-63-specific deubiquitinase BRCC36 promotes NLRP3 deubiquitination and facilitates NLRP3 activation. Previous in vitro study showed that NLRP3 inflammasome is activated by pathologic SNCA. In this work, we revealed that the degeneration of NLRP3 through CMA is an essential event for the regulation of NLRP3 inflammasome. Our study demonstrated that MAPK14-TFEB pathway regulates NLRP3 inflammasome in microglia which is involved in the pathological process of PD. Our data are in line with the previous study demonstrating the protective effect of MAPK14 inhibition and TFEB in PD models ${ }^{11,22}$. Several studies revealed that MAPK14 activation is closely connected to autophagy dysfunction. Mao and colleagues identified that MAPK14 regulates macroautophagy and CMA via phosphorylating ULK and LAMP2A respectively ${ }^{23,24}$. Chen et al. reported that MAPK14 inhibition promotes mitophagy ${ }^{11}$. And recent studies have shown that autophagy plays a key role in inflammasome regulation ${ }^{25}$. Notably, autophagy dysfunction disrupts cell homeostasis thus induces excessive activation of inflammasomes. This study provided evidence showing that MAPK14/TFEB-mediated CMA regulating the NLRP3 inflammasome. Although we identified the role of MAPK14 in activation of NLRP3 inflammasome in SNCA ${ }^{\mathrm{A} 53 \mathrm{~T}}$ mice, the mechanism may be applicable in other neurodegenerative diseases.

\section{Conclusion}


In conclusion, we uncovered a novel NLRP3 degradation pathway by CMA in microglia, which is negative regulation by MAPK14 through direct phosphorylating TFEB at ser211, inhibits the transcription of autophagy gene. Moreover, MAPK14 inhibitor SB203580 provided protection via enhancing TFEBmediated CMA and reducing NLRP3 inflammasome activation both in vitro and vivo. Given that neuroinflammatory response is deeply involved in neurodegenerative diseases, the mechanisms we identified here shed new light on the neurodegenerative process. In addition, the MAPK14-TFEB mediated CMA may be a potential therapeutic target for PD.

\section{Abbreviations}

PD Parkinson's disease

SNpc substantia nigra pars compacta

SNCA a-synuclein

TFEB Transcription Factor EB

CMA chaperone-mediated autophagy

MAPKs Mitogen-activated protein kinases

TLR toll-like receptor

A $\beta$ beta-amyloid

CASP1 caspase 1

AD Alzheimer's disease

ALP autophagy-lysosomal pathway

\section{Declarations}

\section{Ethics approval and consent to participate}

This study was approved by the ethics committee of Southerin Medical University.

\section{Consent for publication}

All authors have approved of the contents of this manuscript and provided consent for publication.

\section{Availability of data and materials}

All data in this study are included in this published article and its supplementary information files. 


\section{Competing interests}

The authors declare that they have no competing interests.

\section{Funding}

This work was supported by the National Natural Science Foundation of China (No.31370763 and No. 81671860).

\section{Author contributions}

JC, KM, HY, YW, HS, LN, HL,CG,YR and XW conducted the experiments. JC, WL,FZ wrote the paper and designed the experiments. All authors reviewed the results and approved the final version of the manuscript.

\section{References}

[1] Karpenko MN, Vasilishina AA, Gromova EA, Muruzheva ZM, Bernadotte A, Interleukin-1 beta, interleukin1 receptor antagonist, interleukin-6, interleukin-10, and tumor necrosis factor-alpha levels in CSF and serum in relation to the clinical diversity of Parkinson's disease, CELL IMMUNOL 2018; 327: 7782;PMID:29478949;10.1016/j.cellimm.2018.02.011

[2] Martinon F, Burns K, Tschopp J, The inflammasome: a molecular platform triggering activation of inflammatory caspases and processing of prolL-beta, MOL CELL 2002; 10: 417-26;PMID:12191486;

[3] Lamkanfi M, Dixit VM, Mechanisms and functions of inflammasomes, CELL 2014; 157: 101322;PMID:24855941;10.1016/j.cell.2014.04.007

[4] Lee E, Hwang I, Park S, Hong S, Hwang B, Cho Y, et al., MPTP-driven NLRP3 inflammasome activation in microglia plays a central role in dopaminergic neurodegeneration, CELL DEATH DIFFER 2018PMID:29786072;10.1038/s41418-018-0124-5

[5] Garcez ML, Mina F, Bellettini-Santos T, Da LA, Schiavo GL, Macieski J, et al., The Involvement of NLRP3 on the Effects of Minocycline in an AD-Like Pathology Induced by beta-Amyloid Oligomers Administered to Mice, MOL NEUROBIOL 2018PMID:30051350;10.1007/s12035-018-1211-9

[6] Lee JC, Kumar S, Griswold DE, Underwood DC, Votta BJ, Adams JL, Inhibition of p38 MAP kinase as a therapeutic strategy, Immunopharmacology 2000; 47: 185-201;PMID:10878289;

[7] Bachstetter AD, Xing B, de Almeida L, Dimayuga ER, Watterson DM, Van Eldik LJ, Microglial p38alpha MAPK is a key regulator of proinflammatory cytokine up-regulation induced by toll-like receptor (TLR) ligands or beta-amyloid (Abeta), J Neuroinflammation 2011; 8: 79;PMID:21733175;10.1186/1742-2094-879 
[8] He Y, She H, Zhang T, Xu H, Cheng L, Yepes M, et al., p38 MAPK inhibits autophagy and promotes microglial inflammatory responses by phosphorylating ULK1, J CELL BIOL 2018; 217: 31528;PMID:29196462;10.1083/jcb.201701049

[9] Munoz L, Ralay RH, Roy SM, Hu W, Craft JM, McNamara LK, et al., A novel p38 alpha MAPK inhibitor suppresses brain proinflammatory cytokine up-regulation and attenuates synaptic dysfunction and behavioral deficits in an Alzheimer's disease mouse model, J Neuroinflammation 2007; 4:

21;PMID:17784957;10.1186/1742-2094-4-21

[10] Wu R, Chen H, Ma J, He Q, Huang Q, Liu Q, et al., c-Abl-p38alpha signaling plays an important role in MPTP-induced neuronal death, CELL DEATH DIFFER 2016; 23: 542-

52;PMID:26517532;10.1038/cdd.2015.135

[11] Chen J, Ren Y, Gui C, Zhao M, Wu X, Mao K, et al., Phosphorylation of Parkin at serine 131 by p38 MAPK promotes mitochondrial dysfunction and neuronal death in mutant A53T alpha-synuclein model of Parkinson's disease, CELL DEATH DIS 2018; 9: 700;PMID:29899409;10.1038/s41419-018-0722-7

[12] Keil E, Hocker R, Schuster M, Essmann F, Ueffing N, Hoffman B, et al., Phosphorylation of Atg5 by the Gadd45beta-MEKK4-p38 pathway inhibits autophagy, CELL DEATH DIFFER 2013; 20: 32132;PMID:23059785;10.1038/cdd.2012.129

[13] Schnöder L, Hao W, Qin Y, Liu S, Tomic I, Liu X, et al., Deficiency of Neuronal p38a MAPK Attenuates Amyloid Pathology in Alzheimer Disease Mouse and Cell Models through Facilitating Lysosomal Degradation of BACE1, J BIOL CHEM 2016; 291: 2067-79;10.1074/jbc.M115.695916

[14] Mehto S, Jena KK, Nath P, Chauhan S, Kolapalli SP, Das SK, et al., The Crohn's Disease Risk Factor IRGM Limits NLRP3 Inflammasome Activation by Impeding Its Assembly and by Mediating Its Selective Autophagy, MOL CELL 2019; 73: 429-45;PMID:30612879;10.1016/j.molcel.2018.11.018

[15] Hong S, Beja-Glasser VF, Nfonoyim BM, Frouin A, Li S, Ramakrishnan S, et al., Complement and microglia mediate early synapse loss in Alzheimer mouse models, SCIENCE 2016; 352: 7126;PMID:27033548;10.1126/science.aad8373

[16] Phan JA, Stokholm K, Zareba-Paslawska J, Jakobsen S, Vang K, Gjedde A, et al., Early synaptic dysfunction induced by alpha-synuclein in a rat model of Parkinson's disease, Sci Rep 2017; 7: 6363;PMID:28743955;10.1038/s41598-017-06724-9

[17] Joshi N, Singh S, Updates on immunity and inflammation in Parkinson disease pathology, J NEUROSCI RES 2018; 96: 379-90;PMID:29072332;10.1002/jnr.24185

[18] Lim S, Kim HJ, Kim DK, Lee SJ, Non-cell-autonomous actions of alpha-synuclein: Implications in glial synucleinopathies, PROG NEUROBIOL 2018; 169: 158-

71;PMID:30173732;10.1016/j.pneurobio.2018.06.010 
[19] De Luca C, Colangelo AM, Alberghina L, Papa M, Neuro-Immune Hemostasis: Homeostasis and Diseases in the Central Nervous System, FRONT CELL NEUROSCI 2018; 12 :

459;PMID:30534057;10.3389/fncel.2018.00459

[20] Kim C, Ho DH, Suk JE, You S, Michael S, Kang J, et al., Neuron-released oligomeric alpha-synuclein is an endogenous agonist of TLR2 for paracrine activation of microglia, NAT COMMUN 2013; 4:

1562;PMID:23463005;10.1038/ncomms2534

[21] Fellner L, Irschick R, Schanda K, Reindl M, Klimaschewski L, Poewe W, et al., Toll-like receptor 4 is required for alpha-synuclein dependent activation of microglia and astroglia, GLIA 2013; 61: 34960;PMID:23108585;10.1002/glia.22437

[22] Ren Y, Chen J, Wu X, Gui C, Mao K, Zou F, et al., Role of c-Abl-GSK3beta Signaling in MPP+-Induced Autophagy-Lysosomal Dysfunction, TOXICOL SCI 2018; 165: 232-

43;PMID:30165626;10.1093/toxsci/kfy155

[23] He Y, She H, Zhang T, Xu H, Cheng L, Yepes M, et al., p38 MAPK inhibits autophagy and promotes microglial inflammatory responses by phosphorylating ULK1, J CELL BIOL 2018; 217: 31528;PMID:29196462;10.1083/jcb.201701049

[24] Li W, Zhu J, Dou J, She H, Tao K, Xu H, et al., Phosphorylation of LAMP2A by p38 MAPK couples ER stress to chaperone-mediated autophagy, NAT COMMUN 2017; 8: 1763;PMID:29176575;10.1038/s41467017-01609-x

[25] Hua F, Li K, Shang S, Wang F, Hu Z, Immune Signaling and Autophagy Regulation, ADV EXP MED BIOL 2019; 1206: 551-93;PMID:31777003;10.1007/978-981-15-0602-4_26

[26] Giasson BI, Duda JE, Quinn SM, Zhang B, Trojanowski JQ, Lee VM, Neuronal alpha-synucleinopathy with severe movement disorder in mice expressing A53T human alpha-synuclein, NEURON 2002; 34: 52133;PMID:12062037;

[27] Ogawa N, Hirose Y, Ohara S, Ono T, Watanabe Y, A simple quantitative bradykinesia test in MPTPtreated mice, Res Commun Chem Pathol Pharmacol 1985; 50: 435-41;PMID:3878557;

[28] Chen J, Ren Y, Gui C, Zhao M, Wu X, Mao K, et al., Phosphorylation of Parkin at serine 131 by p38 MAPK promotes mitochondrial dysfunction and neuronal death in mutant A53T alpha-synuclein model of Parkinson's disease, CELL DEATH DIS 2018; 9: 700;PMID:29899409;10.1038/s41419-018-0722-7

\section{Figures}



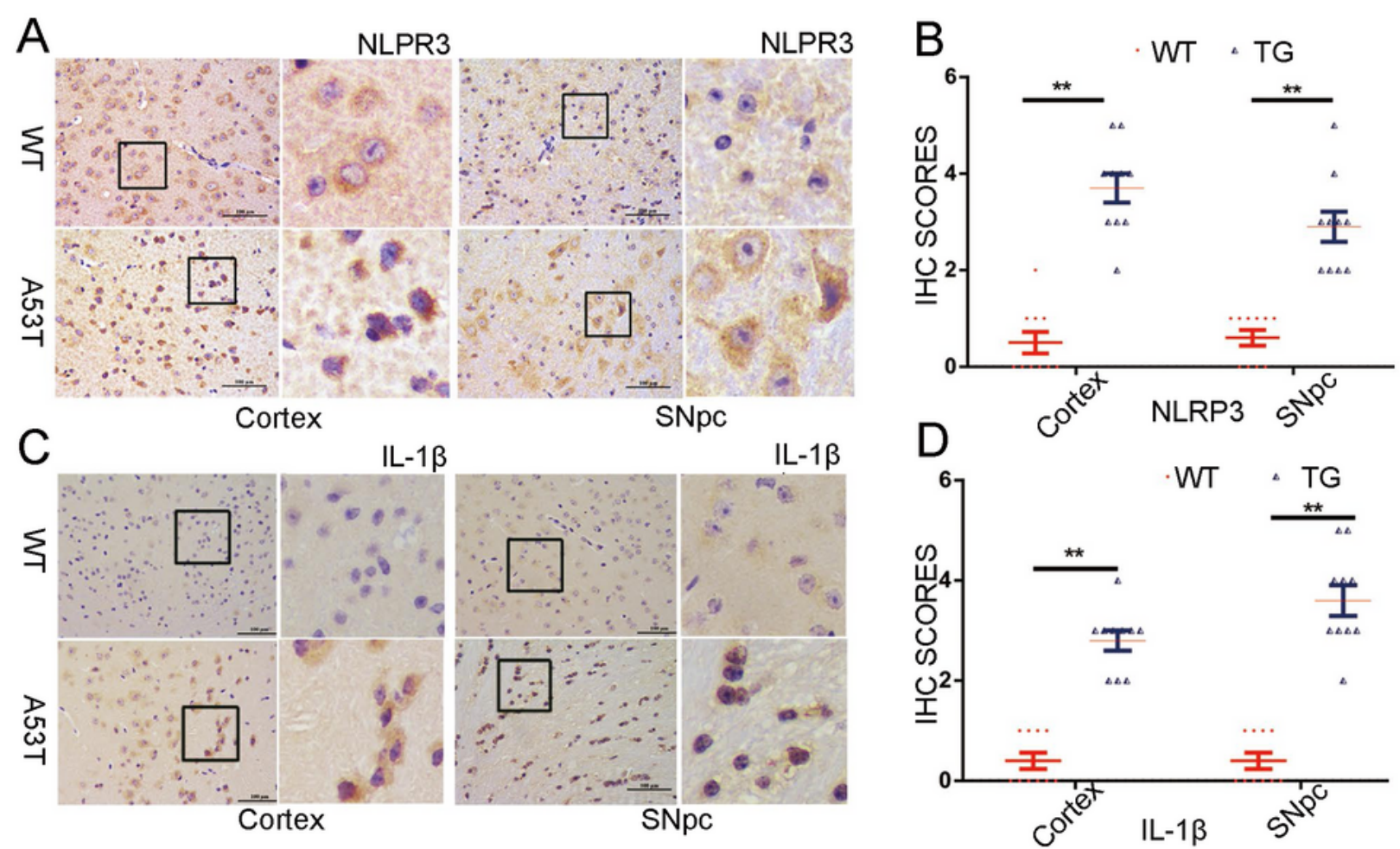

$E$
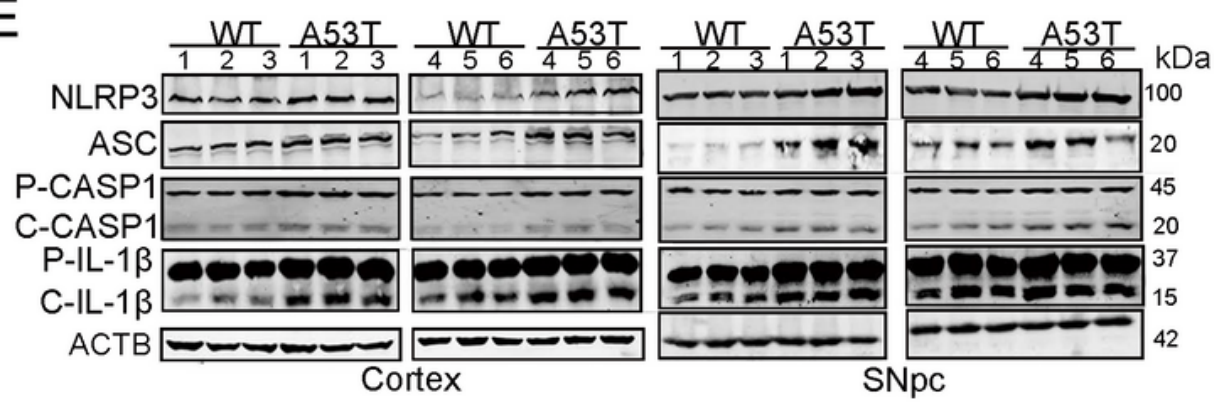

$\mathrm{F}$
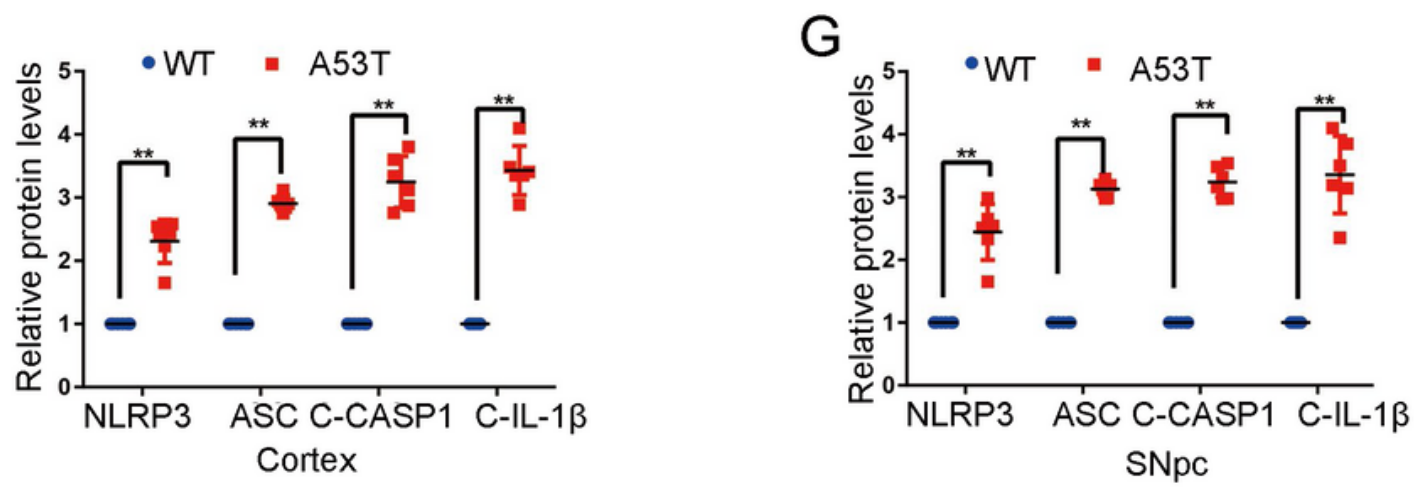

\section{Figure 1}

NLRP3 inflammasome is activated in the SNCAA53T-tg mice (A) Immunohistochemistry (IHC) demonstrating increased NLRP3 protein levels in the cortex and SNpc of 9 months old SNCAA53T-tg mice. (B) Statistical analysis of the scores of NLRP3 staining between SNCAA53T-tg and wild type mice. ${ }^{*} \mathrm{p}<0.05$ (Student's t-test). (C) IHC demonstrating increased IL-1 $\beta$ protein levels in the cortex and SNpc of 9 months old SNCAA53T-tg mice. (D) Statistical analysis of the scores of IL-1 $\beta$ staining between 
SNCAA53T-tg and wild type mice. ${ }^{*} p<0.05$ (Student's t-test). (E-G) Cell lysates from the cortex and SNpc of 9 months old SNCAA53T-tg or wild type mice were immunoblotted. The protein levels of NLRP3, ASC, cleaved CASP1, IL-1 $\beta$ were statistically analyzed in F and G. Mean \pm SEM, $n=6,{ }^{*}<<0.05$ (Student's ttest).

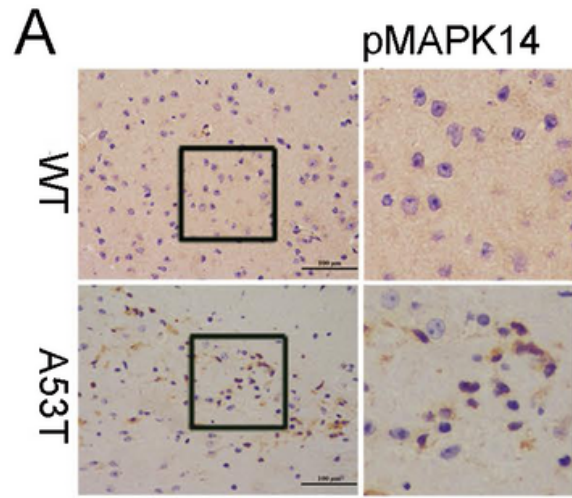

Cortex
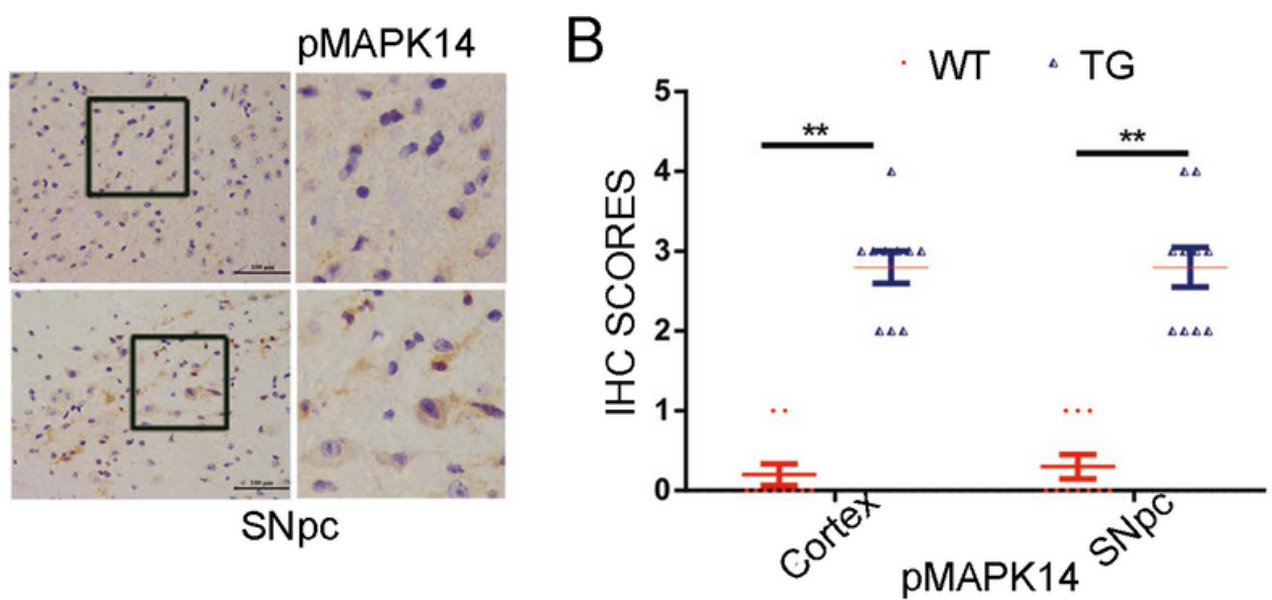

C

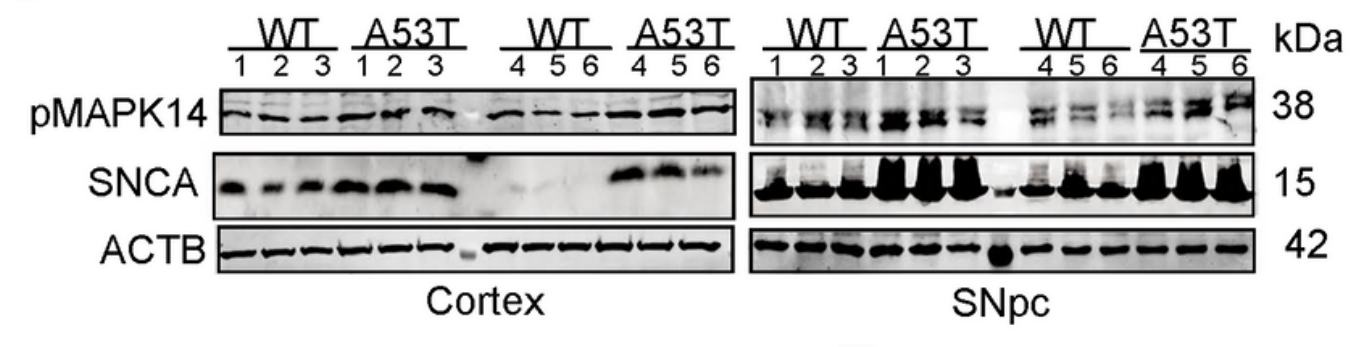

D

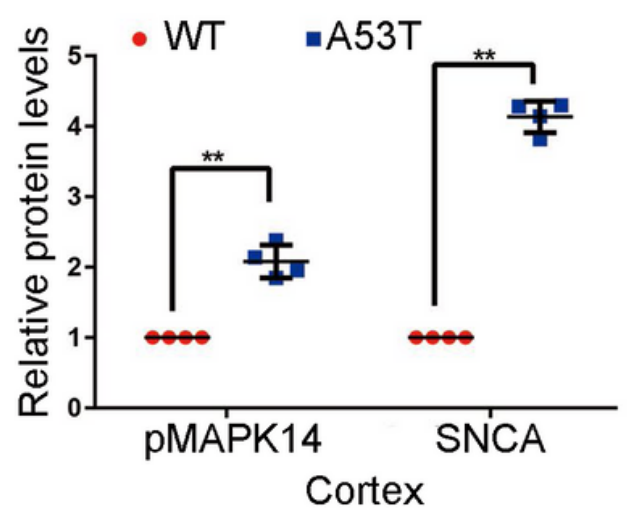

E

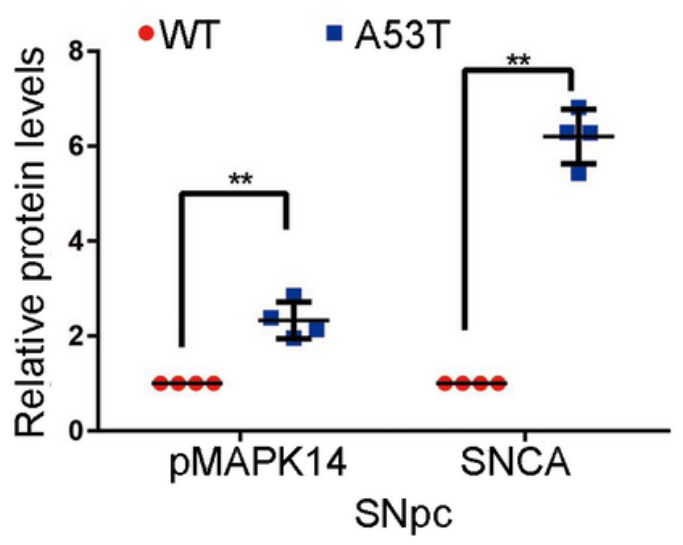

\section{Figure 2}

MAPK14 is activated in both the SNpc and cortex of the SNCAA53T-tg mice (A) IHC demonstrating increased phosphorylated MAPK14 levels in the cortex and SNpc of 9 months old SNCAA53T-tg mice. (B) Statistical analysis of the scores of phosphorylated MAPK14 between SNCAA53T-tg and wild type mice. ${ }^{*} \mathrm{p}<0.05$ (Student's t-test). ( $C, D$ and $E$ ) Lysates from the cortex and SNpc of mice were immunoblotted using the indicated antibodies. The protein levels of phosphorylated MAPK14 and SNCA were statistically analyzed in $D$ and $E$. Mean $\pm S E M, n=6,{ }^{\star} p<0.05$ (Student's t-test). 


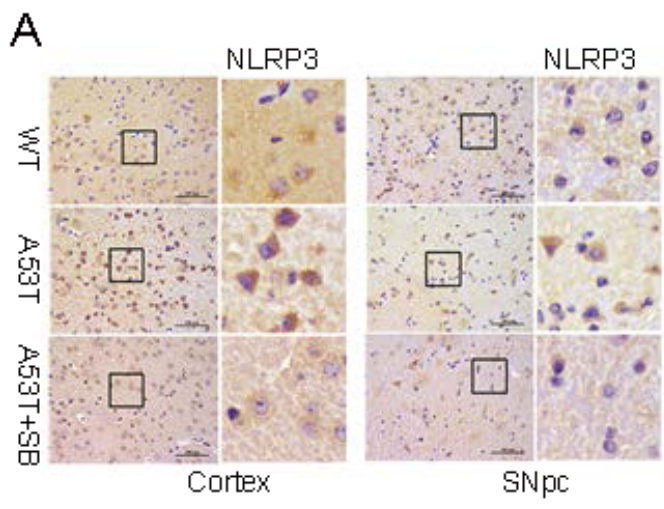

C
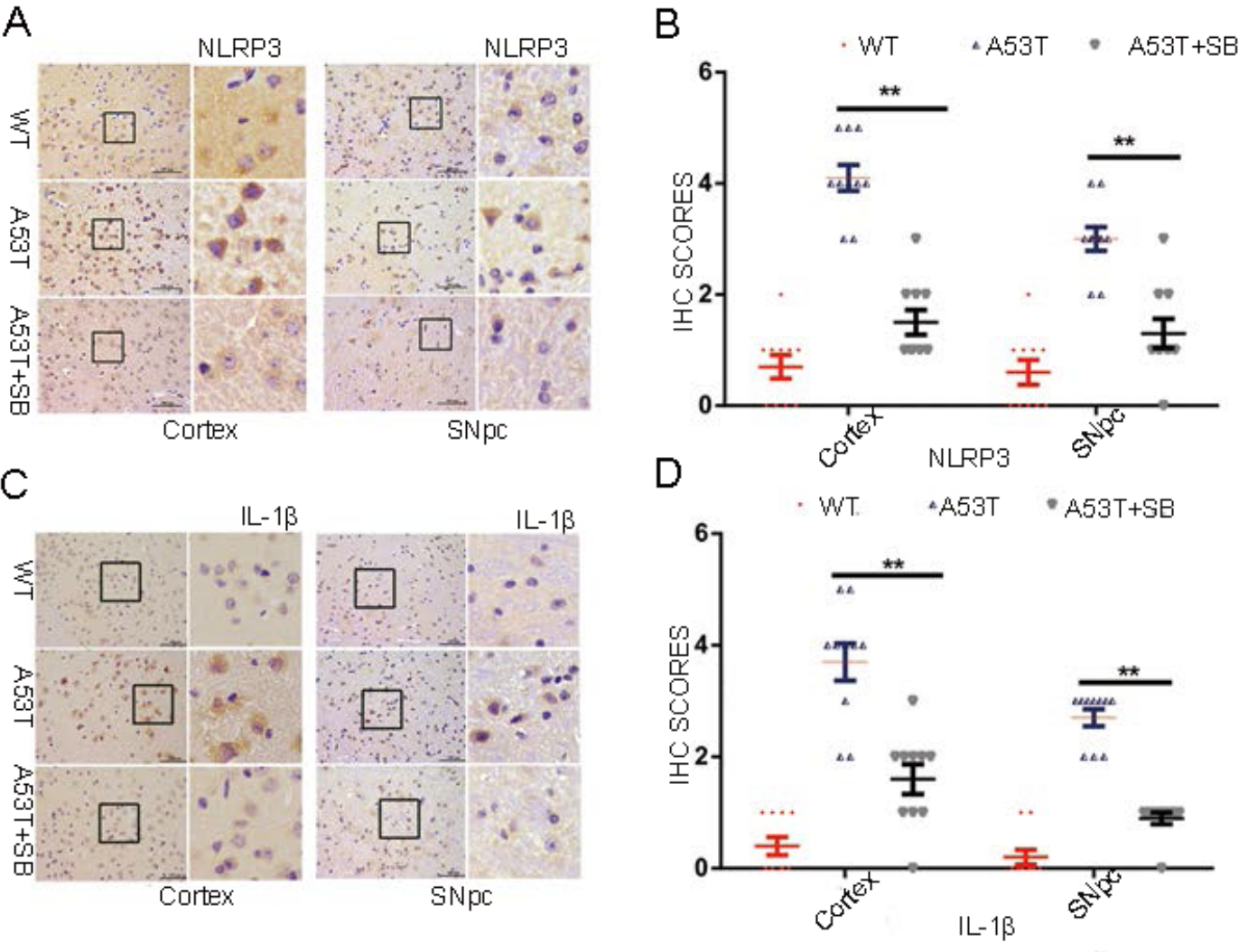

E
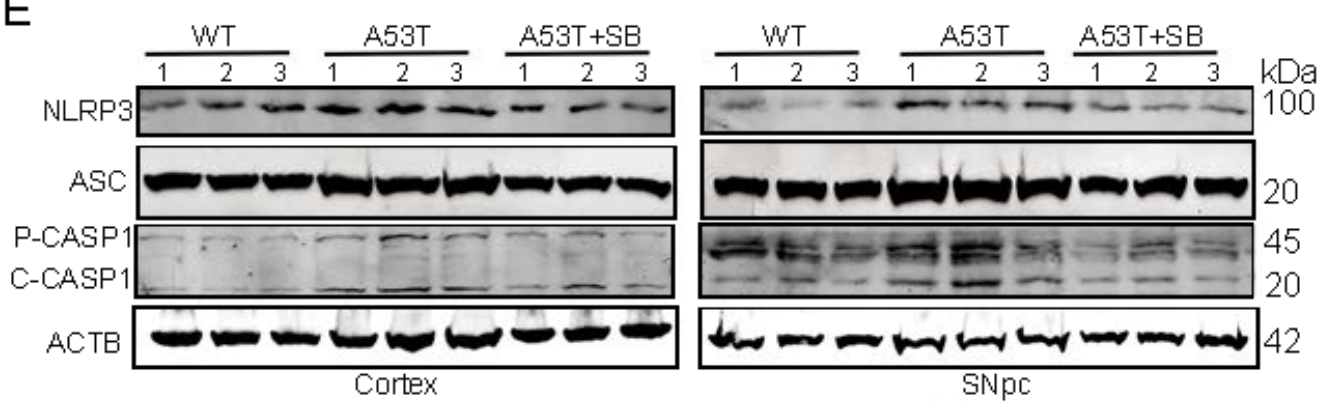

F
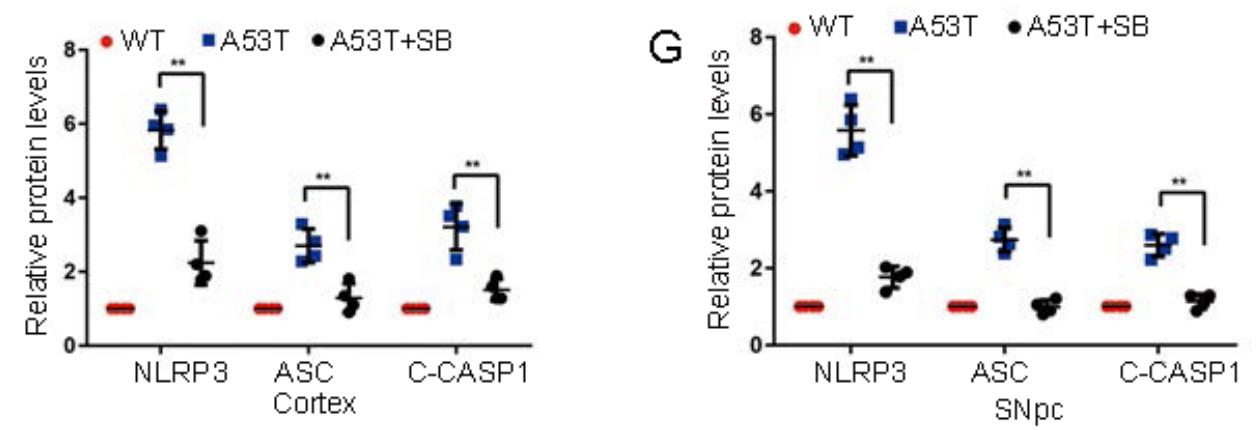

\section{Figure 3}

MAPK14 inhibitor SB203580 inhibits the activation of NLRP3 inflammasome (A) IHC demonstrating SB203580 reduced NLRP3 protein levels in the cortex and SNpc of 9 months old SNCAA53T-tg mice. (B) Statistical analysis of the scores of NLRP3 staining between SNCAA53T-tg and wild type mice. ${ }^{\star} p<0.05$. (C) IHC demonstrating SB203580 reduced IL-1 $\beta$ protein levels in the cortex and SNpc of 9 months old SNCAA53T-tg mice. (D) Statistical analysis of the scores of IL-1 $\beta$ staining between SNCAA53T-tg and wild 
type mice. ${ }^{\star} p<0.05$. $(E, F, G)$ Cell lysates from the cortex and SNpc of mice were immunoblotted using the indicated antibodies. The protein levels of NLRP3, ASC, cleaved CASP1 were statistically analyzed in F and G. ${ }^{*} \mathrm{p}<0.05$.
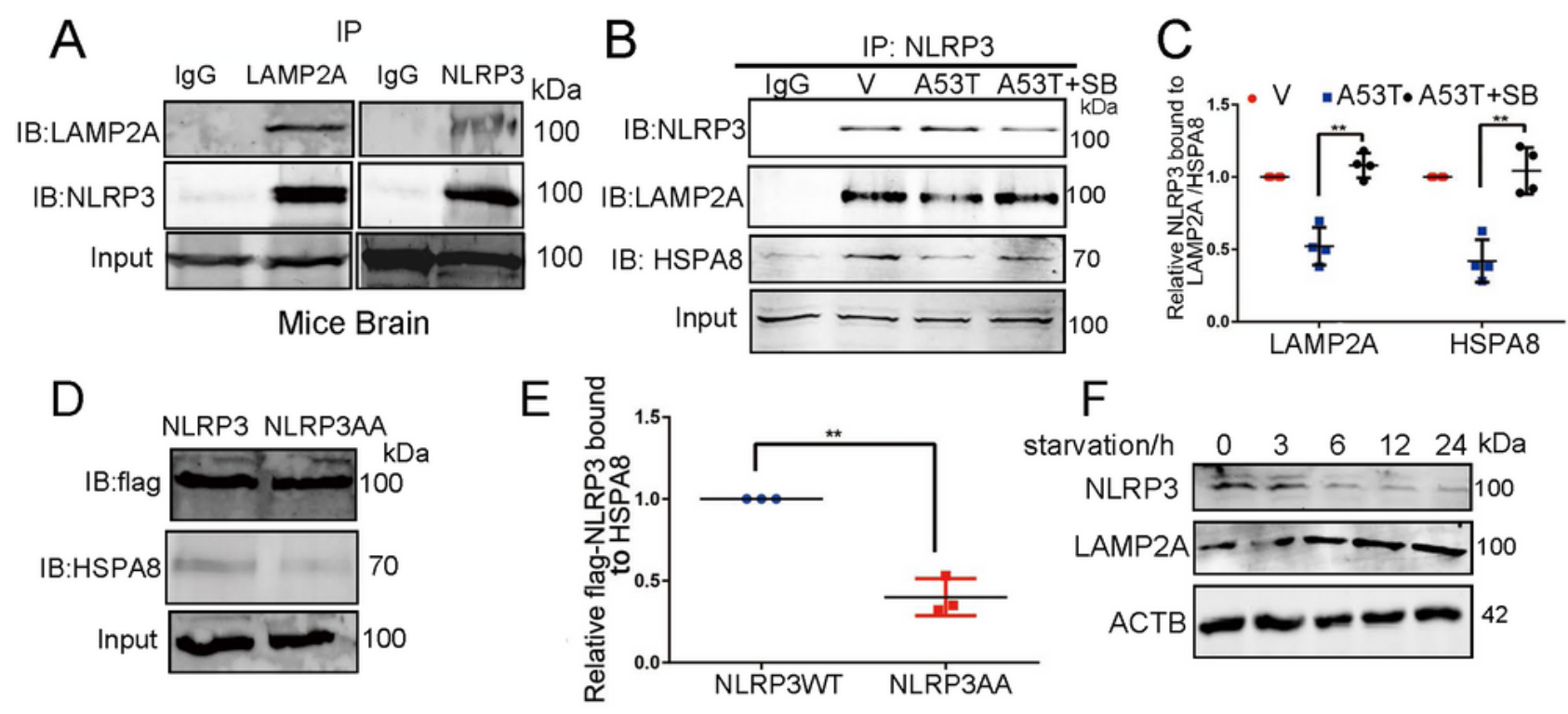

$\mathrm{F}$
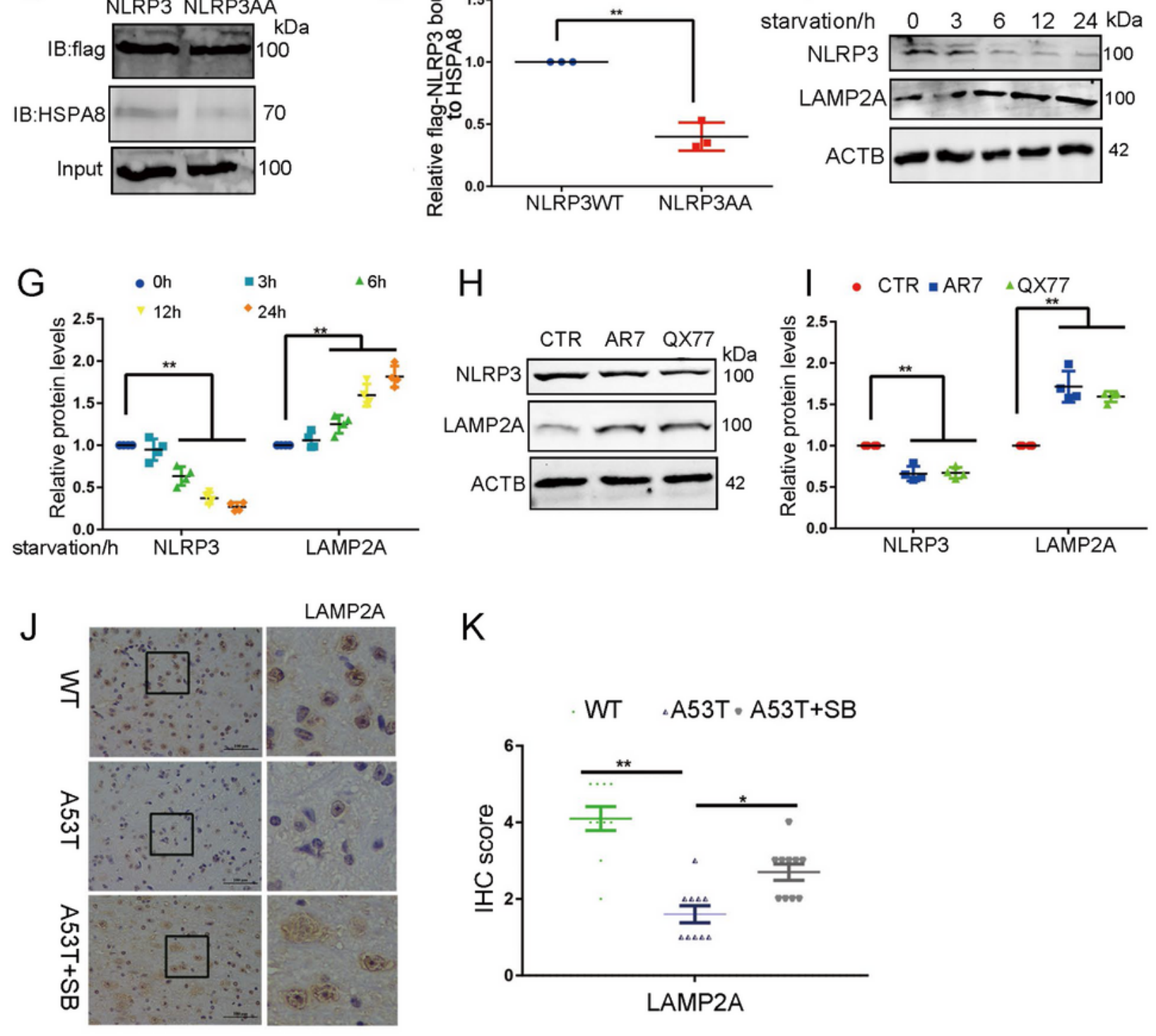

\section{Figure 4}

NLRP3 is degraded through CMA (A) Brain lysates from mice were used for IP using anti-NLRP3 or antiLAMP2A antibody. (B, C) Cell lysates from BV2 cells were used for IP with NLRP3. SB203580 increased 
the NLRP3/LAMP2A and NLRP3/ HSPA8 interaction and showed in C. (D, E) Cell lysates from BV2 cells were used for IP with anti-Flag antibody. Wild type NLRP3-Flag but not NLRP3AA-Flag was coimmunoprecipitated with HSPA8. $(F, G)$ Cell lysates from BV2 cells were immunoblotted to determine the levels of LAMP2A and NLRP3 after starvation. The protein levels of LAMP2A and NLRP3 were statistically analyzed in I and J. Mean $\pm S E M, n=3,{ }^{*} p<0.05$. $(H, I)$ Cell lysates from BV2 cells were immunoblotted to determine the levels of LAMP2A and NLRP3 after treatment with AR7 and QX77. The protein levels of LAMP2A and NLRP3 were statistically analyzed in L. Mean $\pm S E M, n=3$, *p $<0.05$. $(J, K)$ IHC demonstrating decreased LAMP2A protein levels were rescued by SB203580 in the SNpc of SNCAA53T-tg mice. Statistical analysis of the scores of LAMP2A staining were shown in $N .{ }^{*} p<0.05$. 

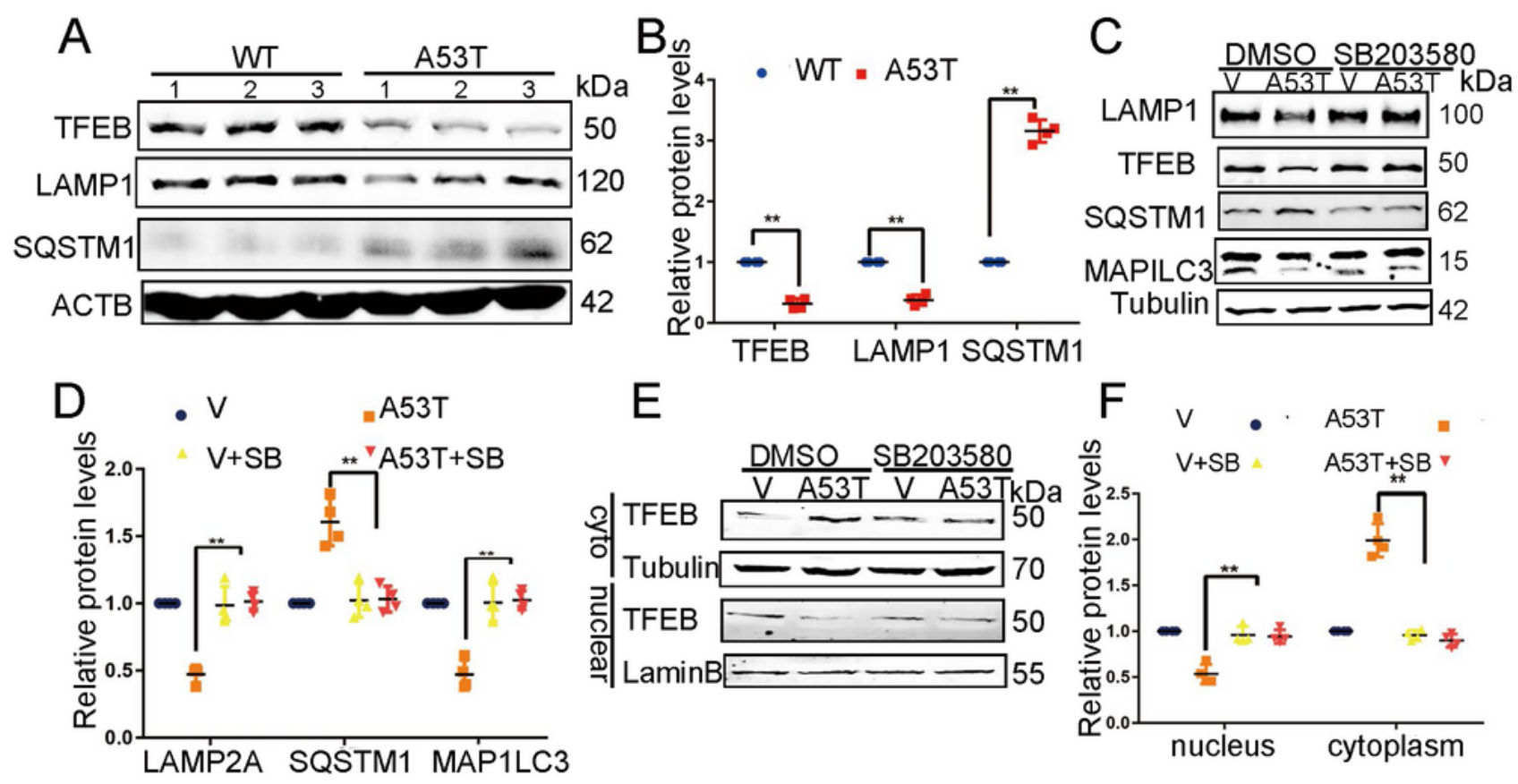

G
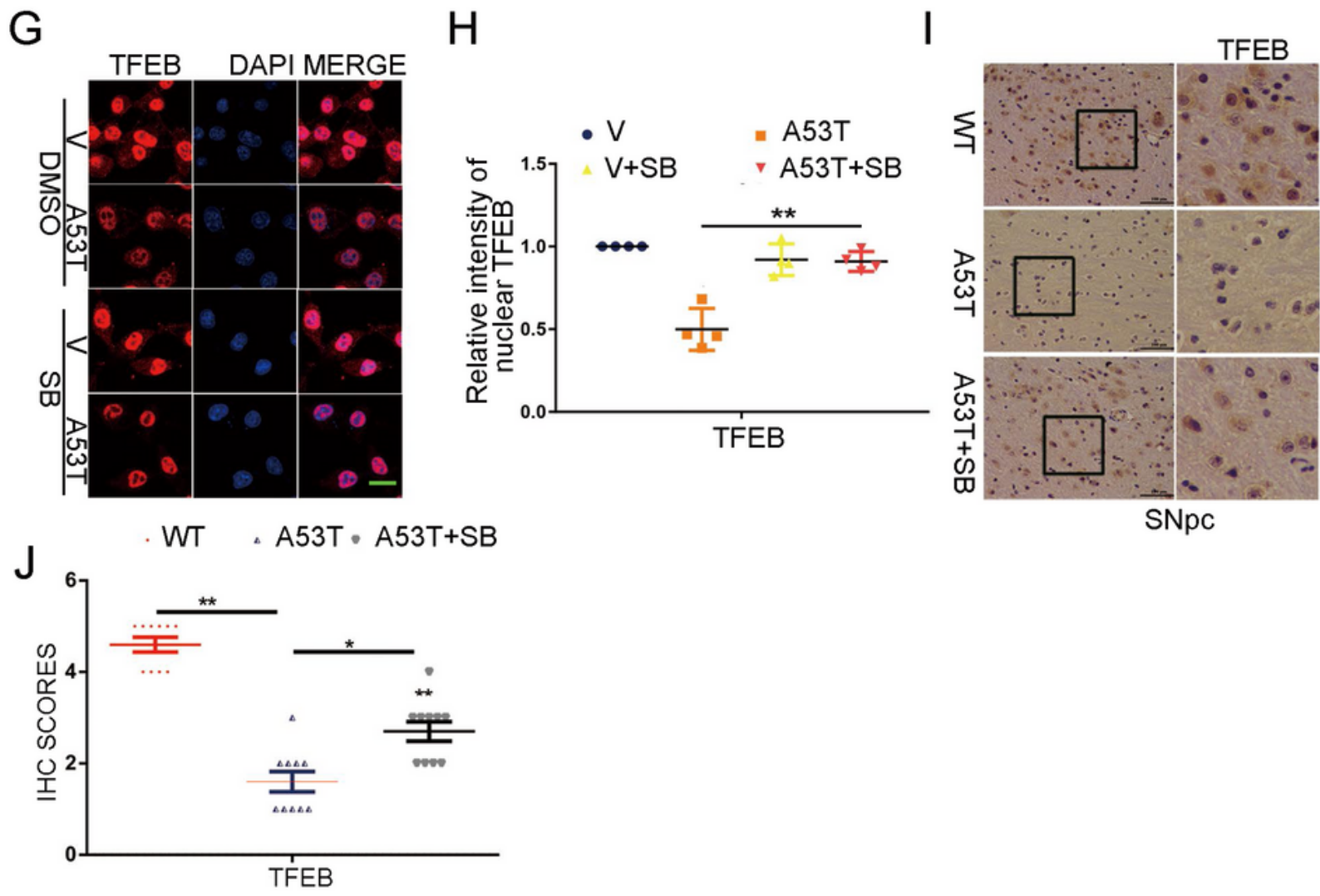

$\mathrm{SNpc}$

Figure 5

SB203580 activates autophagy through TFEB (A, B) Lysates from the SNpc of mice were immunoblotted using the indicated antibodies. The protein levels of TFEB, LAMP1 and SQSTM1 were statistically analyzed in B. Mean $\pm S E M, n=6,{ }^{*}<0.05$. (C, D) Cell lysates from BV2 cells were immunoblotted to determine the levels of LAMP1, MAP1LC3, and SQSTM1 and presented in D. Mean $\pm S E M, n=3, * p<$ 0.05. (E, F) BV2 cells were subjected to subcellular fractionation to determine nuclear translocation TFEB 
in SNCA A53T BV2 cells and presented in $F,{ }^{*} p<0.05$. $(G, H)$ Subcellular localization of TFEB was analyzed by confocal microscopy and presented in $\mathrm{H}$. ${ }^{\star} \mathrm{p}<0.05$. $(\mathrm{I}, \mathrm{J}) \mathrm{IHC}$ demonstrating decreased TFEB protein levels were rescued by SB203580 in the SNpc of SNCAA53T-tg mice. Statistical analysis of the scores of TFEB staining were shown in $\mathrm{J}$. * $\mathrm{p}<0.05$.
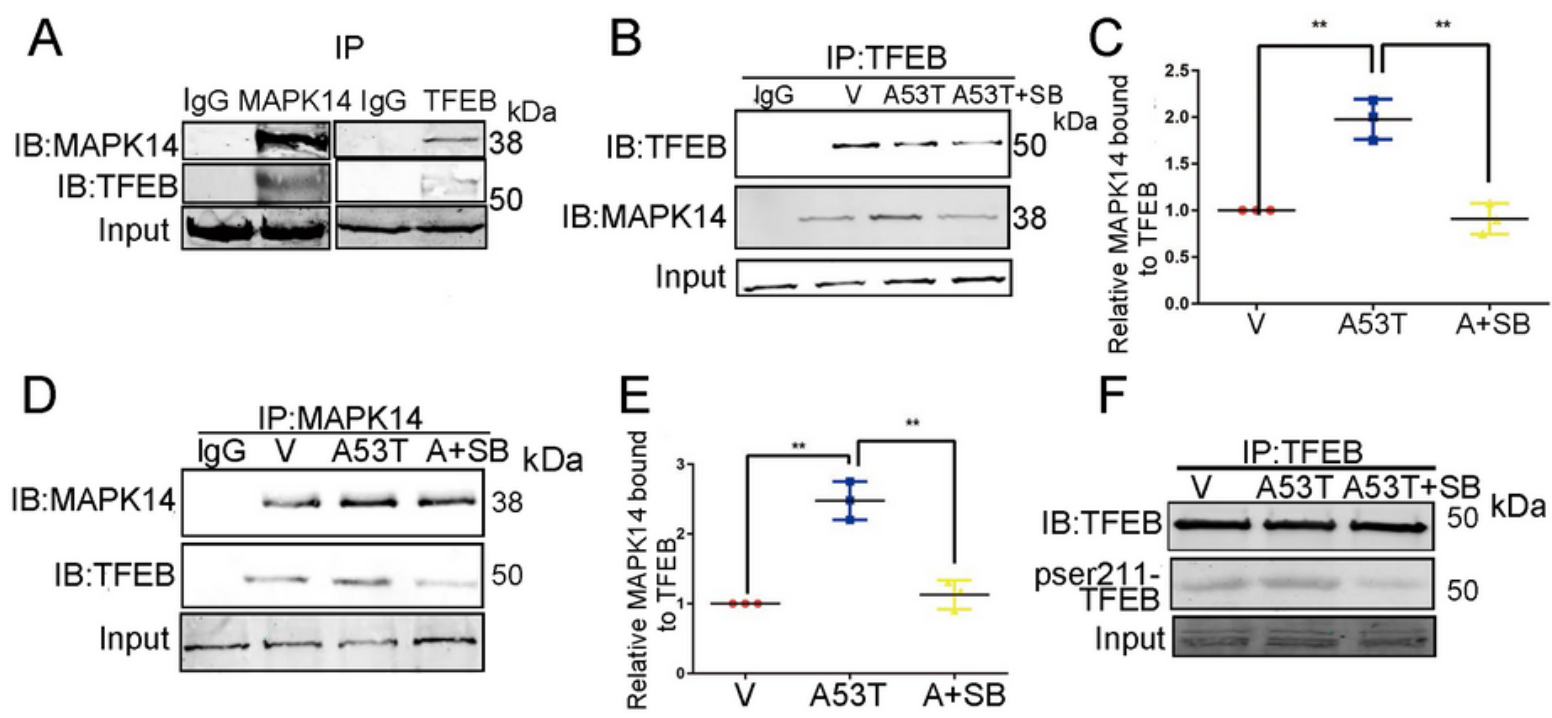

$\mathrm{F}$
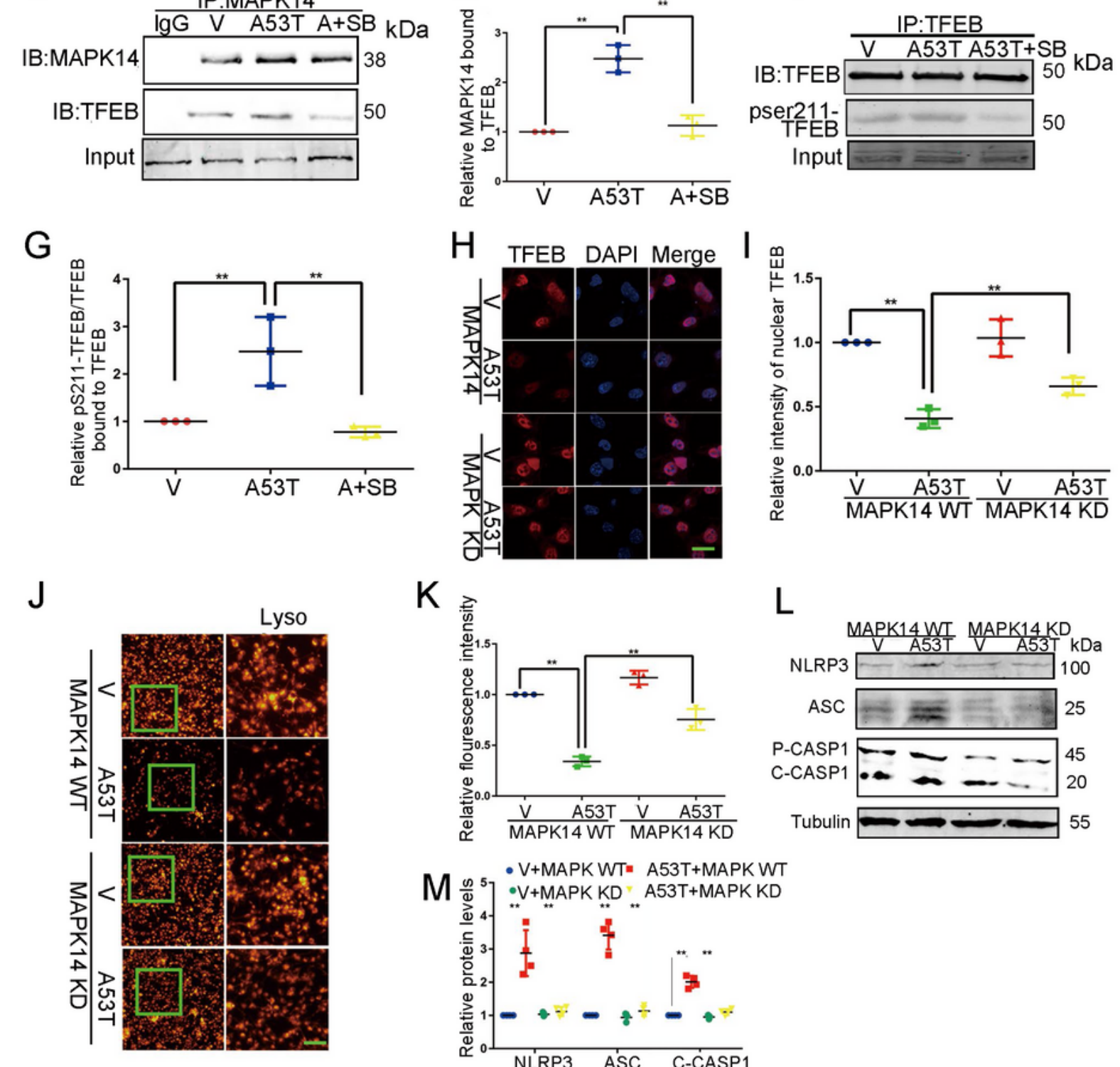

$\mathrm{K}$
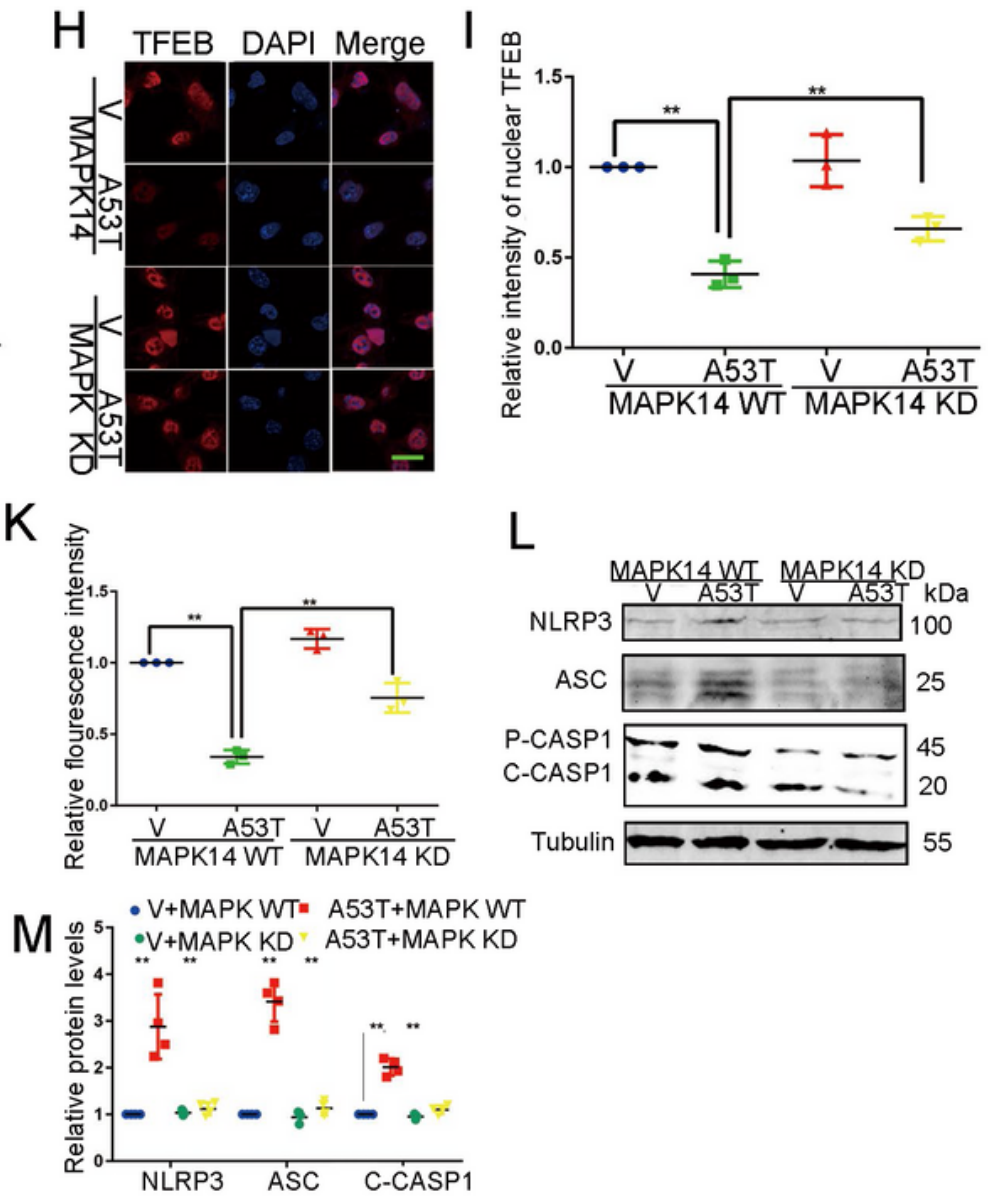

Figure 6 
MAPK14 interacts with and phosphorylates TFEB at serine 211 (A) Lysates from mouse brain were used for IP with anti-TFEB antibody or anti-MAPK14 antibody. $(B, C)$ Cell lysates from BV2 cells were used for IP with anti-TFEB antibody. SB203580 reduced the TFEB/MAPK14 interaction. ${ }^{*} p<0.05$. (D, E) Cell lysates from BV2 cells were used for IP with anti-MAPK14 antibody. SB203580 reduced the TFEB/MAPK14 interaction and shown in E. Mean \pm SEM, $n=3$. ${ }^{*} p<0.05 .(F, G)$ Cell lysates from BV2 cells were used for IP with anti-GFP antibody. SB203580 reduced the TFEB-GFP/14-3-3 interaction. Mean \pm SEM, $n=3 .{ }^{*} p<0.05(\mathrm{H}, \mathrm{l})$. Subcellular localization of TFEB was analyzed by confocal microscopy and shown in I. Mean $\pm S E M, n=3$, ${ }^{\star} p<0.05$. (J,K) BV2 cells were labeled with Lysosome tracker and visualized lysosome biogenesis under a microscope and shown in $K$. Mean $\pm S E M, n=10,{ }^{*}<0.05$. ( $L$, M) Cell lysates from BV2 cells were immunoblotted to determine the levels of NLRP3, ASC and cleaved CASP1 and shown in M. Mean $\pm S E M, n=3,{ }^{*} p<0.05$. 

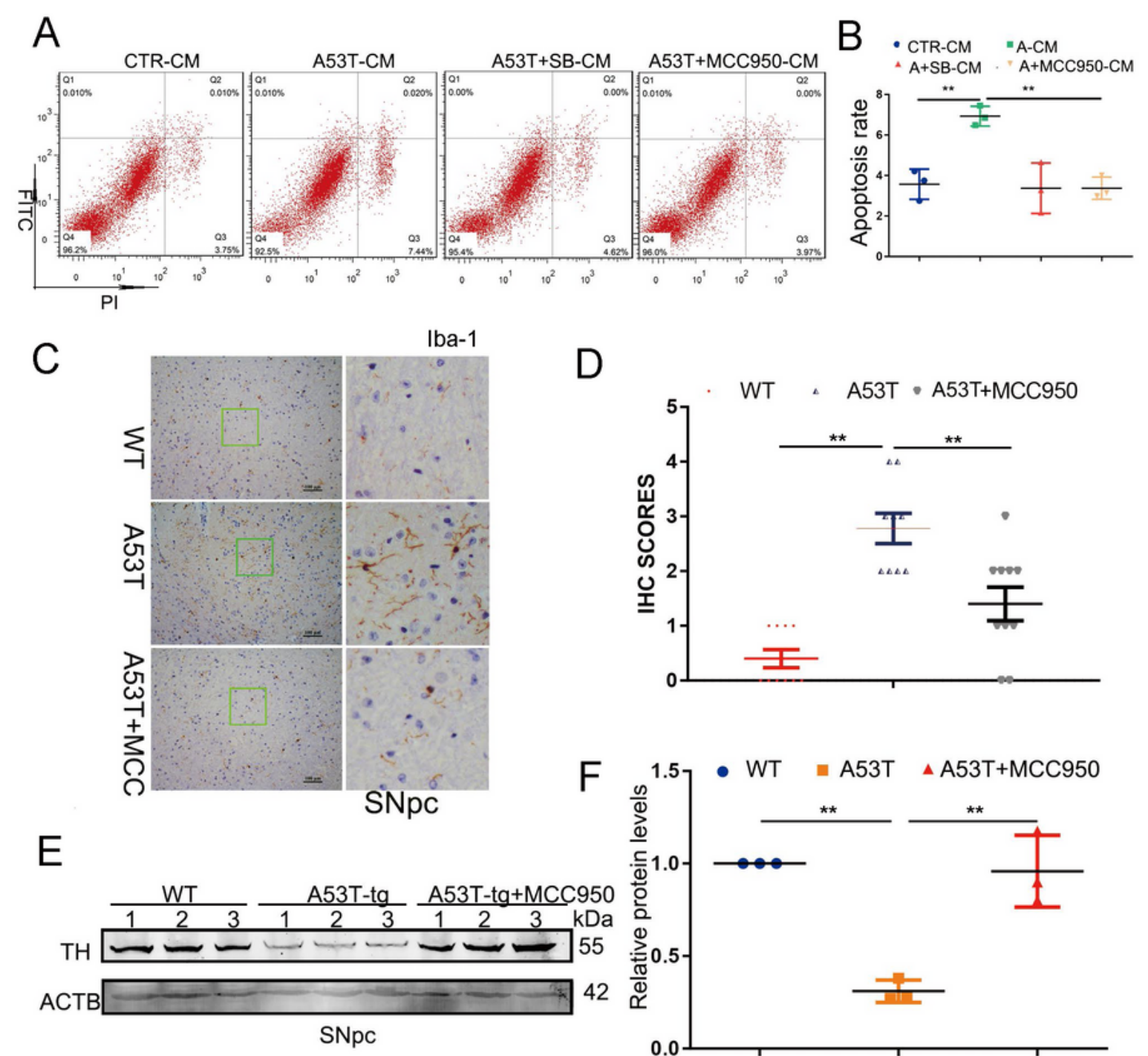

G

$\mathrm{H}$
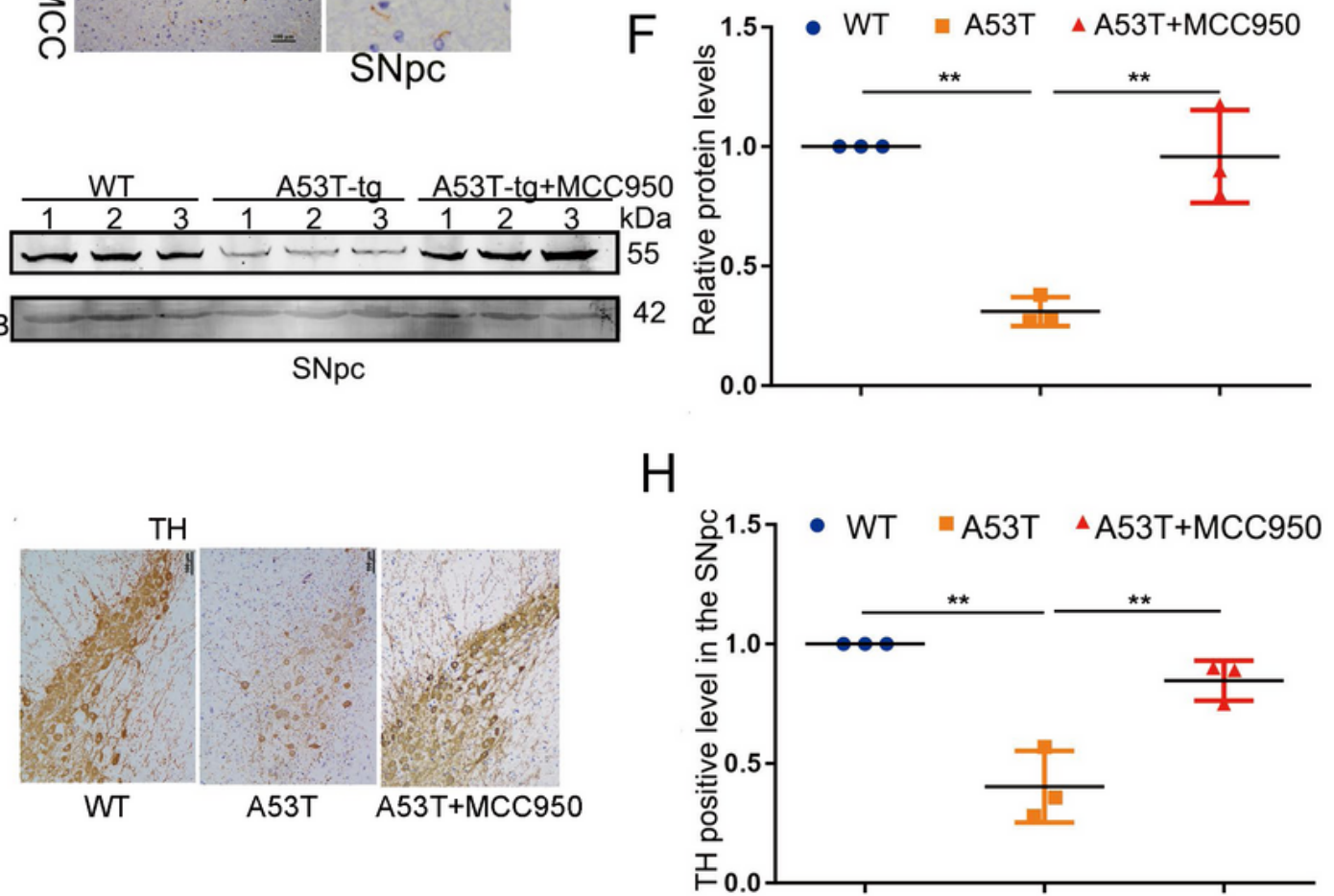

\section{Figure 7}

SNCA-induced NLRP3 activation in microglia promoted dopaminergic neuronal loss (A, B) Cell apoptosis of SN 4741 cells was detected by flow cytometry dyeing with Annexin V-FITC/PI, followed by the treatment with conditioned medium from BV2. Mean $\pm S E M, n=3$, * $p<0.05$. (C, D) Immunohistochemistry (IHC) demonstrating the levels of IBA-1 in the SNpc of 9 months of wide type, SNCA A53T-tg and SNCA A53T-tg mice treated with MCC950. Statistical analysis of the scores of IBA-1 
staining were shown in D. Mean $\pm S E M, n=3 .{ }^{*} p<0.05$. (E, F) Cell lysates from the SNpc of mice were immunoblotted using the indicated antibodies and shown in F. Mean $\pm S E M, n=6$, * $p<0.05$. $(G, H)$ Immunohistochemistry (IHC) demonstrating the levels of TH in the SNpc of 9 months of wide type, SNCA A53T-tg and SNCA A53T-tg mice treated with MCC950. Statistical analysis of the levels of TH staining were shown in $\mathrm{H}$. Mean $\pm \mathrm{SEM}, \mathrm{n}=3$. ${ }^{*} \mathrm{p}<0.05$.
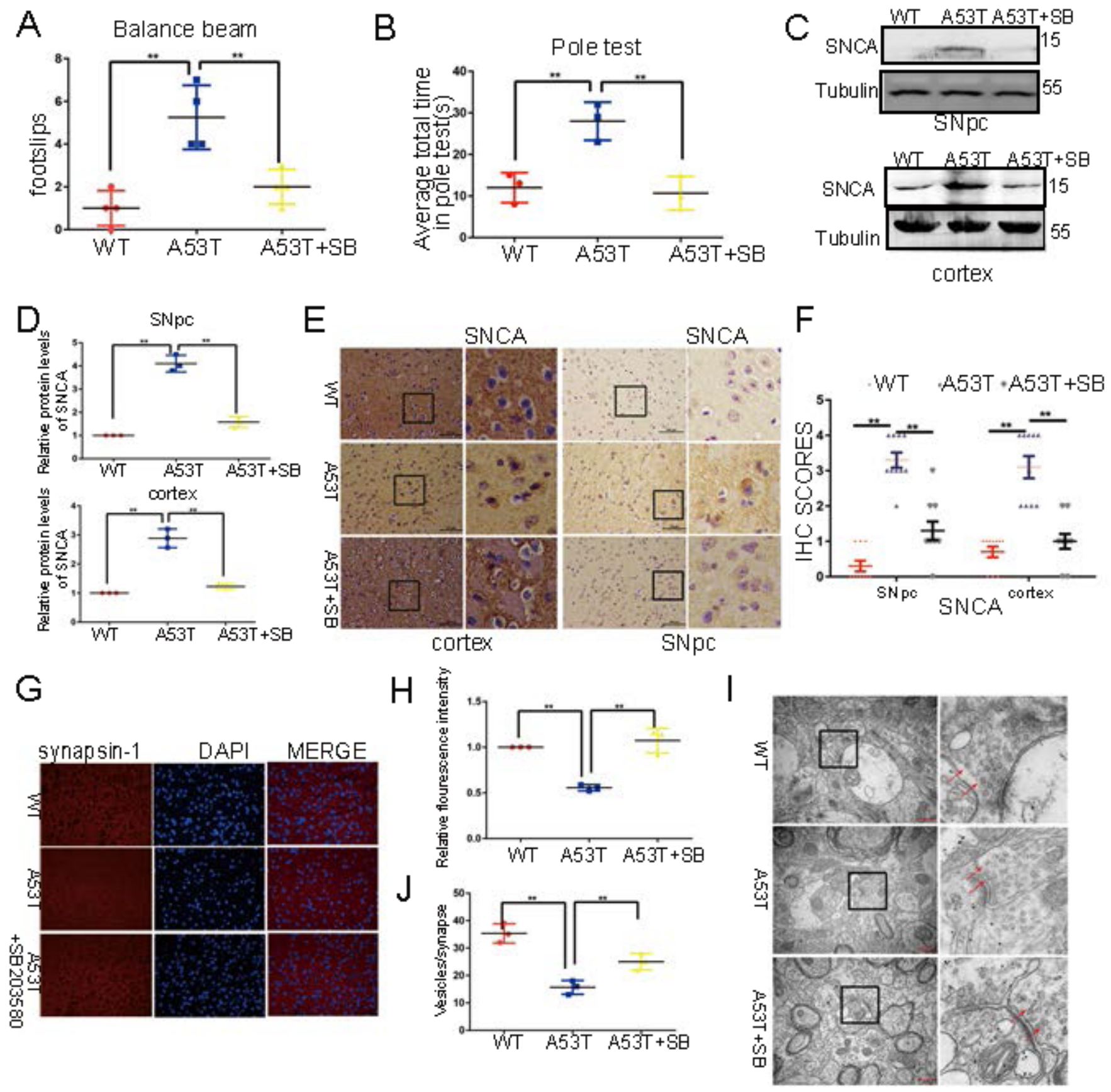

Figure 8

SB203580 plays a protective role in the SNCAA53T-tg mice (A) Balance beam foot slips were quantified after PBS or SB203580 injection. (B) Results of Pole test were quantified after PBS or SB203580 injection. (C, D) Cell lysates from the SNpc and cortex of mice were immunoblotted using the SNCA 
antibody and shown in D. Mean \pm SEM, $n=6,{ }^{*} p<0.05$. (E, F) IHC staining of SNCA in the SNpc and

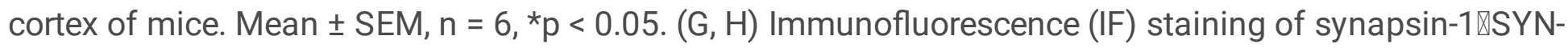
1 \in the SNpc of mice. Mean \pm SEM, $n=6,{ }^{\star} p<0.05$. $(I, J)$ TEM was used to examine the number of synapses and shown in $\mathrm{J}$ as mean \pm SEM. $n=3 .{ }^{*} p<0.05$. The provided Scale bar in merge image represent $500 \mathrm{~nm}$.

\section{Supplementary Files}

This is a list of supplementary files associated with this preprint. Click to download.

- GR.jpg

- S1.tif

- S2.tif

- S3.tif

- S4.tif

- S5.tif

- S6.tif

- S7.tif 